\title{
La actividad de los participantes como fuente de información para promover la colaboración. Una analítica del aprendizaje basada en el modelo de Influencia Educativa Distribuida
}

\section{The participants' activity as a source of information to promote collaboration. A learning analytic based on the Distributed Educational Influence model}

\author{
César Coll \\ Universitat de Barcelona, Barcelona, España \\ ccoll@ub.edu \\ Anna Engel \\ Universitat de Barcelona, Barcelona, España \\ anna.engel@ub.edu \\ Shamaly Niño \\ Universitat de Barcelona, Barcelona, España \\ shamaly.nino@gmail.com
}

\section{RESUMEN}

Este trabajo presenta la versión inicial de una analítica del aprendizaje inspirada en el modelo de influencia educativa distribuida (IED). La idea central del modelo de IED es que en las situaciones de trabajo y aprendizaje colaborativo todos los participantes son fuentes potenciales de ayuda para los otros participantes. Sobre esta base, se ha llevado a cabo una investigación con un triple objetivo: analizar el impacto de la información proporcionada a los participantes sobre el proceso colaborativo y su evolución, indagar si la información proporcionada tiene un efecto diferencial en función de su naturaleza y contrastar la utilidad de la analítica desarrollada para analizar los procesos de colaboración en línea. Para ello, se han seguido cuatro grupos de estudiantes que participan en una serie de foros en línea en el marco de una asignatura de máster. Los resultados indican que en general la información proporcionada a los participantes sobre su actividad tiene un impacto en el proceso colaborativo. No ha sido posible, en cambio, demostrar que la naturaleza de la información proporcionada tenga un impacto diferencial sobre el proceso de colaboración. Los resultados también han puesto de manifiesto algunas limitaciones de los indicadores en que se concreta la analítica desarrollada.

PALABRAS CLAVE: analíticas del aprendizaje, aprendizaje colaborativo, foros en línea, influencia educativa, influencia educativa distribuida. 


\begin{abstract}
This article introduces the initial version of a learning analytic based in the model of distributed educational influence (DEI). The central idea of the DEI model is that in collaborative learning situations all the participants are potential sources of support for other participants. On this basis, a research was carried out with a triple objective: i) to analyze if the information provided to the participants has an impact on their collaborative process, ii) to determine whether the provided information has a differential effect depending on the nature of its content and iii) to contrast the usefulness of the analytic developed to analyze the processes of online collaboration. For this purpose, four groups of master's degree students participating in a series of online forums were studied. In general, the results indicate that the information provided to the participants about their activity has an impact on their collaborative processes. However, it has not been possible to demonstrate any differential impact on their collaboration processes depending on the nature of the provided information. The results also point out some limitations on the indicators of the learning analytic proposed.
\end{abstract}

KEYWORDS: collaborative learning, distributed educational influence, educational influence, learning analytics, online forums.

\title{
Introducción
}

Uno de los retos más importantes a los que se enfrentan en la actualidad las instituciones de educación formal es ayudar a los estudiantes a adquirir las competencias necesarias para aprender a aprender a lo largo y lo ancho de la vida (Banks et al., 2007). Entre estas nuevas competencias cabe destacar la capacidad para aprender en redes digitales. De acuerdo con Wilson y Scalise (2015), el uso de los medios digitales para aprender y el aprendizaje a través de las redes digitales serán cada vez más importantes para el desarrollo personal y profesional. Ser capaz de colaborar, compartir y construir conocimiento en contextos y situaciones diferentes y con una diversidad de personas con las que no se puede interactuar cara a cara será un elemento clave para desenvolverse eficazmente en la sociedad del siglo XXI (Jenkins, Clinton, Purushotma, Robinson y Weigel, 2006; Binkley et al., 2012). La capacidad para participar en actividades colaborativas es vista cada vez más como una competencia básica que las instituciones educativas deben cultivar con el fin de preparar a los estudiantes "para convertirse en participantes de valiosas prácticas sociales de construcción colectiva de conocimiento" (Roschelle, 2013, p. 67).

En la última década se han desarrollado diferentes líneas de investigación centradas en la utilización de las tecnologías digitales para apoyar y guiar los procesos interactivos entre estudiantes y enseñarles a participar, comunicarse, colaborar y aprender a través de la colaboración. Entre ellas ocupan un lugar destacado el campo

La actividad de los participantes como fuente de información para promover la colaboración. Una analítica del aprendizaje basada en el modelo de Influencia Educativa Distribuida. César Coll, Anna Engel y Shamaly Niño.

Página 2 de 36. 
de investigación y diseño conocido como analíticas del aprendizaje -Learning Analytics-. La Society for Learning Analytics Research define este campo de estudio como "la medición, recolección, análisis y presentación de datos sobre los alumnos y sus contextos, con el propósito de comprender y optimizar el aprendizaje y los entornos en los que se produce" (Siemens y Long, 2011, p. 34). Suthers y Verbert (2013), por su parte, lo definen como un campo que se sitúa a medio camino entre las ciencias del aprendizaje, la investigación educativa y el uso de técnicas computacionales para capturar y analizar datos.

En este marco, la finalidad de nuestra investigación es desarrollar una analítica del aprendizaje con una sólida base teórica y empírica que permita proporcionar información en tiempo real a los participantes sobre las características centrales del proceso de colaboración y aprendizaje que están llevando a cabo (Xing, Guo, Petakovic y Goggins, 2015). El desarrollo de una analítica del aprendizaje que pueda contribuir de forma decisiva a mejorar el aprendizaje colaborativo en línea requiere diseñar unos procedimientos de cálculo capaces de procesar la información recopilada sobre la actividad de los aprendices y su contexto de aprendizaje. Sin embargo, como puntualizan tanto Brown (2012) como Suthers y Verbert (2013) y Ferguson (2012), este diseño requiere disponer de un modelo teórico potente que, más allá de una aplicación mecánica o instrumentalista (Zapata-Ros, 2013), ayude a decidir cuál es la información relevante que hay que recopilar y procesar, cómo hay que interpretarla y cómo generar, a partir de los resultados obtenidos, actuaciones dirigidas a mejorar los procesos y los resultados del aprendizaje colaborativo. De acuerdo con esta llamada de atención, nuestro planteamiento consiste en desarrollar una analítica del aprendizaje basada en el modelo de influencia educativa distribuida (Coll, Bustos, y Engel, 2015)

Esta analítica debe cumplir, además, las tres exigencias siguientes. En primer lugar, debe posibilitar un análisis de los datos de alto nivel que permita definir e identificar perfiles diferenciados de la actividad de los estudiantes establecidos a partir de la combinación de indicadores e índices de distinto tipo (Dawson, Gašević, Siemens y Joksimovic, 2014; Dawson y Siemens, 2014; Haya, Daems, Malzahn, Castellanos y Hoppe, 2015; Rodríguez-Triana, Martínez-Monés, Asensio-Pérez y Dimitriadis, 2015). En segundo lugar, debe permitir presentar la información a los estudiantes en un formato de visualización sencillo que no requiera conocimientos especializados para comprenderla y usarla con el fin de mejorar la propia actividad y el proceso colaborativo (Fidalgo-Blanco, Sein-Echaluce, García-Peñalvo y Conde, 2015; Mazza y Dimitrova, 2007; Romero, Ventura y García, 2007; Valentín y Burgos, 2015). Y, en tercer lugar, debe ser lo suficientemente flexible para ser utilizada en una gama amplia de entornos de aprendizaje en línea en distintos tipos de actividades con objetivos y contenidos educativos diferentes y con grupos de estudiantes de distintas características.

La actividad de los participantes como fuente de información para promover la colaboración. Una analítica del aprendizaje basada en el modelo de Influencia Educativa Distribuida. César Coll, Anna Engel y Shamaly Niño.

Página 3 de 36. 
En este trabajo presentamos la versión inicial de la analítica desarrollada, su aplicación a un proceso de aprendizaje colaborativo en línea y los resultados obtenidos. Para ello, presentamos en primer lugar las líneas directrices del modelo de IED y un conjunto de indicadores sobre la actividad de los participantes considerados relevantes para la mejora del proceso de colaboración que se derivan de este modelo. Seguidamente, mostramos una aplicación de la métrica propuesta -es decir, del conjunto de indicadores- a la actividad desplegada por cuatro grupos de estudiantes universitarios que llevan a cabo una tarea colaborativa en un entorno en línea. A continuación, presentamos los resultados sobre el impacto que la entrega de información relativa a la actividad de los participantes tiene en la evolución de la calidad del proceso colaborativo de los cuatro grupos de estudiantes. Finalmente, discutimos estos resultados y sus implicaciones para la mejora de la analítica del aprendizaje propuesta.

\section{Marco teórico}

El concepto de influencia educativa (IE), elaborado en el marco de una serie de trabajos sobre el análisis de las prácticas educativas en contextos presenciales (ver, por ejemplo, Coll, Colomina, Onrubia y Rochera, 1992; Coll, Onrubia y Mauri, 2008; Colomina, Onrubia,y Rochera, 2001), hace referencia a los procesos interpsicológicos mediante los cuales los profesores, y en su caso otros agentes educativos o actores presentes en el contexto de aprendizaje, ayudan a los estudiantes a construir significados progresivamente más ricos, complejos y válidos sobre los contenidos de enseñanza y aprendizaje. Para que la IE ejercida por el profesor sea eficaz debe ser contingente y ajustarse al desarrollo y evolución del proceso de aprendizaje. Así, las ayudas que se entreguen a los estudiantes no pueden ser siempre y en todo momento las mismas, sino que han de ir variando en tipo, cantidad e intensidad en función de los avances y retrocesos que los alumnos experimentan y, a la vez, se deben ir retirando progresivamente a medida que éstos se muestren más autónomos en su aprendizaje (Coll, 2010).

Desde esta perspectiva, la IE se ejerce tanto en las situaciones de construcción guiada como de construcción colaborativa del conocimiento (Mercer, 1995). Sin embargo, una diferencia importante entre estos dos tipos de situaciones tiene que ver con quién ejerce la IE. En el primer caso, la fuente principal de ayudas y apoyos al aprendizaje es el profesor, mientras que en los procesos de construcción colaborativa del conocimiento todos los participantes son fuentes potenciales de ayuda para los otros participantes (Bustos, 2011; Bustos, Coll y Engel, 2009; Coll, Bustos, Engel, de Gispert y Rochera, 2013; Coll y Engel, 2014). El concepto de influencia educativa distribuida (IED) refleja la idea de que en las situaciones colaborativas el ejercicio de la IE se distribuye entre todos los participantes, ya que todos deben y "pueden contribuir mediante sus actuaciones a promover los procesos comunicativos, sociales y cognitivos implicados en el aprendizaje" (Coll, Bustos y Engel, 2011, p. 659).

La actividad de los participantes como fuente de información para promover la colaboración. Una analítica del aprendizaje basada en el modelo de Influencia Educativa Distribuida. César Coll, Anna Engel y Shamaly Niño.

Página 4 de 36. 
En los procesos de aprendizaje colaborativo mediados por ordenador, típicamente basados en la comunicación escrita, asíncrona y sin contacto visual directo, la posibilidad de que los participantes sean capaces de ofrecer y recibir ayudas de manera efectiva depende del cumplimiento de ciertas exigencias. Así, es necesario que los participantes accedan de forma periódica al entorno digital, que lean las contribuciones del resto de los participantes, que publiquen sus propias aportaciones y que establezcan relaciones entre sí a través de lo que se escriben y responden mutuamente a lo largo del proceso colaborativo (Coll, Engel y Bustos, 2009; Engel, Coll y Bustos, 2013). Pero además las contribuciones de los participantes deben ser relevantes para el proceso colaborativo y de aprendizaje. De este modo, un segundo bloque de exigencias se relaciona con la necesidad de que los participantes expliciten las formas en que van organizando la actividad conjunta a lo largo del proceso de aprendizaje en mucho mayor grado de lo que habitualmente es necesario que lo hagan en un contexto presencial. Así, con el fin de garantizar un adecuado desarrollo del proceso de colaboración, los participantes han de ejercer la IE en tres planos o dimensiones de la actividad conjunta: i) la gestión de la participación social (relativa a quién puede hacer o decir qué, cuándo, cómo, dirigiéndose a quién y con qué medios); ii) la gestión de la tarea académica (relativa a qué hay que hacer, cómo hay que hacerlo, mediante qué procedimientos, qué productos hay que generar y con qué características); y iii) la gestión de los significados sobre los contenidos de enseñanza y aprendizaje (relativa a la presentación de información, formulación de dudas, petición de aclaraciones, requerimientos, síntesis, manifestaciones de acuerdo o desacuerdo, etc., sobre los contenidos).

El modelo de IED proporciona un referente teórico apropiado para el diseño de una analítica del aprendizaje orientada a recopilar, procesar e interpretar información sobre los aspectos y dimensiones de la actividad de los participantes asociados al ejercicio de la IE y a su nivel de distribución entre los participantes; una analítica del aprendizaje, por tanto, que vincula el éxito de la colaboración y sus resultados al hecho de que los participantes asuman efectivamente su responsabilidad como dispensadores y receptores de ayuda y se alcance un nivel adecuado de distribución de la IE en el conjunto del grupo (Coll, Bustos y Engel, 2015).

Dos tipos de indicadores permiten identificar y establecer el nivel de ejercicio de IE de los participantes en un proceso de aprendizaje colaborativo y son relevantes, en consecuencia, para valorar la calidad del proceso de colaboración. Por una parte, tenemos los indicadores relacionados con las exigencias antes mencionadas sobre el acceso al entorno digital, la realización de contribuciones, la lectura de las contribuciones de los otros participantes y el establecimiento de relaciones entre sí; por otra, los indicadores sobre el contenido de las contribuciones atendiendo a las tres dimensiones en las que cabe ejercer la IE: la participación social, la tarea académica y los significados sobre los contenidos de aprendizaje. La toma en consideración de

La actividad de los participantes como fuente de información para promover la colaboración. Una analítica del aprendizaje basada en el modelo de Influencia Educativa Distribuida. César Coll, Anna Engel y Shamaly Niño.

Página 5 de 36. 
ambos tipos de informaciones simultáneamente proporciona una visión situada y multidimensional de la actividad de los participantes en un entorno digital que puede ser utilizada por estos para mejorar su propia actividad y los procesos de colaboración en los que están implicados.

En lo que concierne a los primeros, en este trabajo hemos operado con once índices relacionados con el acceso, la participación y la conectividad, de los que seis conciernen a la actividad individual y cinco a la actividad del grupo en su conjunto. Estos índices se definen como sigue.

El índice individual de acceso (IIA) refiere al número de días que un participante accede al entorno en línea en el que se desarrolla la actividad sobre el número total de días de duración de la actividad. La hipótesis es que hay una frecuencia mínima de días de acceso por debajo de la cual es prácticamente imposible seguir el hilo de las contribuciones, ofrecer ayudas al resto de participantes y, por supuesto, ajustar estas ayudas a las necesidades de los otros participantes.

El patrón individual de acceso (PIA) refiere a la continuidad del acceso al entorno en línea. Los participantes que están ausentes del entorno de enseñanza y aprendizaje durante periodos de tiempo largos difícilmente pueden hacer un seguimiento de las contribuciones del resto de participantes y ofrecerles ayudas ajustadas y, por lo tanto, difícilmente pueden ejercer una influencia educativa.

El índice individual de lecturas (IIL) es la proporción de contribuciones leídas por un participante con respecto al total de contribuciones hechas por el resto de participantes. El argumento que justifica la elección de este índice es que, para poder proporcionar ayudas ajustadas y contingentes, es necesario compartir el contexto discursivo que se está construyendo y esto sólo es posible si se leen las contribuciones de los participantes.

El índice individual de contribuciones (IIC) refiere a la proporción de contribuciones escritas por un participante en relación con las requeridas en las directrices establecidas para la realización de la tarea. En este caso, el argumento es que difícilmente pueden ofrecerse ayudas ajustadas contribuyendo menos de lo exigido por esas directrices. Más aún, es razonable argumentar que la posibilidad de ayudar a los otros participantes requiere un número de contribuciones superior al mínimo exigido por las directrices.

El patrón individual de contribuciones (PIC) refleja la distribución de las contribuciones escritas por un participante a lo largo del desarrollo de la actividad. La hipótesis es que los participantes que contribuyen de forma continuada y sostenida durante el desarrollo de la actividad están mejor situados para ejercer la IE que los que no lo hacen así.

La actividad de los participantes como fuente de información para promover la colaboración. Una analítica del aprendizaje basada en el modelo de Influencia Educativa Distribuida. César Coll, Anna Engel y Shamaly Niño.

Página 6 de 36. 
El índice individual de reciprocidad (IIR) es la proporción de relaciones comunicativas recíprocas que establece un participante con otros participantes sobre el total de relaciones recíprocas posibles que podría llegar a establecer. El argumento en este caso es que los participantes que establecen relaciones recíprocas con un mayor número de participantes y que, por lo tanto, ocupan una posición central en la red de relaciones están mejor situados para ejercer la IE que los que ocupan lugares periféricos.

El índice grupal de acceso (IGA) es el sumatorio de los días que los diferentes participantes acceden al entorno en línea sobre los días de duración de la actividad por el número de participantes.

El índice grupal de lecturas (IGL) es el total de contribuciones leídas por todos los participantes sobre el número de lecturas posibles de las contribuciones realizadas por todos los participantes.

El índice grupal de contribuciones (IGC) es el total de contribuciones escritas por todos los participantes sobre el número esperado de contribuciones de todos los participantes de acuerdo con lo establecido en las directrices de desarrollo de la actividad.

El índice de densidad de la red (IDR) se define como la proporción de relaciones comunicativas reales que establecen los participantes con otros participantes sobre el total de relaciones posibles que podrían llegar a establecer [n(n-1)]. En este caso, se trata de captar la amplitud de los vínculos o conexiones que crean los participantes entre sí a partir de sus contribuciones dirigidas a los demás.

El índice grupal de reciprocidad (IGR) es la proporción de pares de participantes que tienen conexión recíproca entre sí. Se calcula dividiendo el número total de díadas recíprocas reales sobre el número total de díadas recíprocas posibles.

Respecto al segundo tipo de indicadores, relacionados con el contenido de las contribuciones de los participantes, hemos definido tres índices de carácter individual relativos a las tres dimensiones asociadas al ejercicio de la IE y tres de carácter grupal:

El índice individual de gestión de la participación social (IIP) refiere a las contribuciones o fragmentos de contribuciones que un participante dedica a las reglas o instrucciones relativas a cómo participar en la actividad -por ejemplo, proponer las reglas de participación que deben regir en el grupo, valorar esas reglas, pedir precisiones sobre ellas, definir los roles que asumirá cada participante, etc.- sobre el total de contribuciones o fragmentos de contribuciones de ese participante.

La actividad de los participantes como fuente de información para promover la colaboración. Una analítica del aprendizaje basada en el modelo de Influencia Educativa Distribuida. César Coll, Anna Engel y Shamaly Niño.

Página 7 de 36. 
El índice individual de gestión de la tarea académica (IIT) refiere a las contribuciones o fragmentos de contribuciones que un participante dedica a las reglas o instrucciones relativas a las características y exigencias de la tarea, los pasos a seguir para realizarla o las características del producto final -por ejemplo, pedir precisiones sobre la tarea, valorar las características de la tarea propuesta, proponer una forma de abordar la tarea, etc.- sobre el total de contribuciones o fragmentos de contribuciones de ese participante.

El índice individual de gestión de los significados sobre los contenidos de aprendizaje (IIS) refiere a las contribuciones o fragmentos de contribuciones que un participante dedica a negociar y discutir los contenidos de enseñanza y aprendizaje -por ejemplo, aportar información, requerir a los otros que se pronuncien sobre lo aportado, pedir precisiones sobre una contribución, identificar errores o lagunas, elaborar síntesis, etc.- sobre el total de contribuciones o fragmentos de contribuciones de ese participante.

El índice grupal de gestión de la participación social (IGP) refiere a las contribuciones o fragmentos de contribuciones que el grupo en su conjunto dedica a las reglas o instrucciones relativas a cómo participar en la actividad sobre el total de contribuciones o fragmentos de contribuciones del conjunto de los miembros del grupo.

El índice grupal de gestión de la tarea académica (IGT) refiere a las contribuciones o fragmentos de contribuciones que el grupo en su conjunto dedica a las reglas o instrucciones relativas a las características y exigencias de la tarea, los pasos a seguir para realizarla o las características del producto final sobre el total de contribuciones o fragmentos de contribuciones del conjunto de miembros del grupo.

El índice grupal de gestión de los significados sobre los contenidos de aprendizaje (IGC) refiere a las contribuciones o fragmentos de contribuciones del grupo en su conjunto relacionados con los contenidos de enseñanza y aprendizaje sobre el total de contribuciones o fragmentos de contribuciones del conjunto de miembros del grupo.

\section{Objetivos}

El presente estudio persigue un triple objetivo:

(i) discernir si el hecho de proporcionar información a los participantes que están desarrollando colaborativamente una tarea sobre cómo ejercen la IE en la misma tiene un impacto en el proceso colaborativo y en su evolución;

(ii) indagar si la naturaleza de la información proporcionada sobre el ejercicio de la IE información estructural sobre acceso, participación y conectividad, información sobre el contenido de las aportaciones, ambos tipos de informaciones simultáneamente-

La actividad de los participantes como fuente de información para promover la colaboración. Una analítica del aprendizaje basada en el modelo de Influencia Educativa Distribuida. César Coll, Anna Engel y Shamaly Niño.

Página 8 de 36. 
tiene un efecto diferencial sobre el proceso colaborativo y su evolución;

(iii) contrastar la utilidad de la analítica del aprendizaje desarrollada a partir del modelo teórico de IED para describir y analizar procesos de trabajo colaborativo en línea, especialmente en lo que concierne a la adecuación y validez de los indicadores que contempla.

\section{Método}

Para alcanzar estos objetivos, hemos utilizado una estrategia metodológica de estudio de casos (Yin, 2006) que goza de una larga tradición en la investigación educativa y psicoeducativa en general, así como en las investigaciones sobre aprendizaje colaborativo mediado por ordenador en particular (ver, por ejemplo, Stahl, Koschmann y Suthers, 2006).

\section{Contexto y participantes}

En el estudio participaron 33 estudiantes (29 mujeres y cuatro hombres) que cursaban una asignatura de carácter obligatorio del Máster Interuniversitario de Psicología de la Educación en la Universidad de Barcelona durante el curso 2013-2014. Los contenidos de enseñanza y aprendizaje se abordaron en ocho sesiones presenciales quincenales, cada una de ellas dedicada a un núcleo temático distinto. Durante el periodo de dos semanas comprendido entre una sesión presencial y la siguiente, los estudiantes, organizados en cuatro grupos formados al azar e integrados por 8 o 9 miembros cada uno, debían elaborar un glosario de algunos conceptos clave del núcleo temático (NT) presentado en la sesión presencial previa, utilizando para ello un foro creado a tal fin en el aula Moodle de la asignatura. Los grupos se mantuvieron estables en las ocho sesiones. De los 14 días previstos para la elaboración del glosario del NT en el foro, los grupos dedicaban la primera semana básicamente a la revisión individual de las lecturas obligatorias y la segunda a elaborar las definiciones de los conceptos propuestos para el glosario. Todos los estudiantes debían realizar al menos cuatro aportaciones al foro cuando su duración era inferior a dos semanas y ocho aportaciones cuando era superior. El número de conceptos propuestos para confeccionar los glosarios fueron los siguientes: NT1: 10; NT2: 7; NT3: 6; NT4: 6; NT5: 5; NT6: 5; NT7: 6 . A título de ejemplo, algunos conceptos propuestos para formar parte de los glosarios fueron "sentido del aprendizaje" (NT1), "plasticidad cerebral" (NT2), "cognición distribuida" (NT3), "comunidad de práctica" (NT4), "motivo de la actividad" (NT5), "sistema de actividad" (NT6), "personalización del aprendizaje" (NT7). Todos los estudiantes estaban familiarizados con la plataforma Moodle y habían desarrollado actividades grupales de distinta naturaleza a través de los foros en diferentes asignaturas durante el semestre anterior.

\section{Diseño}

La actividad de los participantes como fuente de información para promover la colaboración. Una analítica del aprendizaje basada en el modelo de Influencia Educativa Distribuida. César Coll, Anna Engel y Shamaly Niño.

Página 9 de 36. 
Con el fin de apoyar a los estudiantes a mejorar sus procesos de colaboración en línea, al inicio de cada foro de elaboración del glosario se les entregó individualmente información sobre la actividad individual y grupal que habían desarrollado en el foro correspondiente al NT anterior, utilizando para ello la herramienta "tarea" del aula Moodle de la asignatura. De este modo, como se aprecia en la Figura 1, los estudiantes recibieron la primera entrega de información al inicio del foro 2 (F2) y las siguientes entregas al inicio de los restantes foros (F3, F4, F5, F6 y F7).

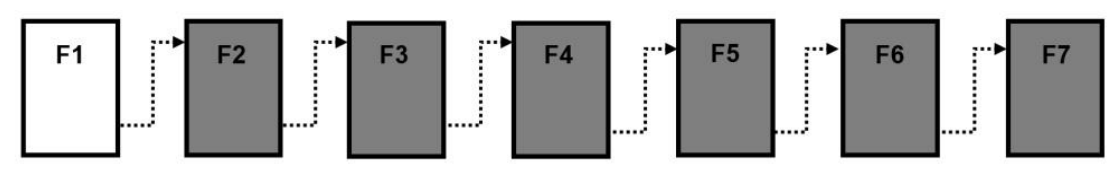

Figura 1. Momento de entrega de la información a los participantes sobre la actividad desarrollada en el foro anterior.

En los siete foros los grupos recibían información diferenciada sobre la actividad grupal e individual desarrollada en el foro anterior. Así, a los estudiantes del grupo 1 G1- se les proporcionaba individualmente información basada en indicadores de tipo estructural sobre acceso, participación y conectividad relativa tanto al grupo en su conjunto como a cada participante individual. Los indicadores se calcularon a partir de los registros de actividad que proporciona de forma automática la plataforma Moodle. En la Tabla 1 se indica el procedimiento utilizado para el cálculo de los indicadores individuales y grupales de acceso, participación y conectividad, de acuerdo con las definiciones presentadas más arriba. Para cada uno de ellos se señala el valor umbral por encima del cual se considera que la actividad del participante o del grupo en su conjunto, según el caso, es teóricamente adecuado para el ejercicio de la IE en el aspecto o dimensión al que remite. Además de los indicadores que se mencionan en la Tabla 1, para cada foro se construyó un sociograma con la ayuda del software UCINET (Borgatti, Everett y Freeman, 2002) que representa las relaciones comunicativas entre los participantes, de manera que a cada participante se le proporcionaba información sobre la posición que ocupaba en el sociograma.

La actividad de los participantes como fuente de información para promover la colaboración. Una analítica del aprendizaje basada en el modelo de Influencia Educativa Distribuida. César Coll, Anna Engel y Shamaly Niño.

Página 10 de 36. 
Tabla 1. Información relacionada con el acceso, la participación y la conectividad proporcionada a los participantes del G1

\section{Indicadores de tipo estructural}

\section{Indicadores individuales de la actividad en el foro} Índice individual de acceso (IIA)

Valor umbral para el ejercicio adecuado de la IE: $\geq 0,50$

Cuando el participante accede al foro al menos en la mitad de los días que dura la actividad en el foro en línea

\section{Patrón individual de acceso (PIC)}

Continuo: cuando el participante no presenta ningún periodo de más de tres días sin acceso durante la última semana de actividad en el foro en línea

Discontinuo: cuando el participante presenta uno o más períodos de más de tres días sin acceso durante la última semana de actividad en el foro en línea

\section{Índice individual de lecturas (IIL)}

Valor umbral para el ejercicio adecuado de la IE: $\geq 0,90$

Cuando el participante lee prácticamente la totalidad de las contribuciones hechas en el foro en línea

\section{Índice individual de contribuciones (IIC) \\ Valor umbral para el ejercicio adecuado de la IE: $\geq 1,50$}

Cuando el participante realiza como mínimo un $50 \%$ más de contribuciones que las exigidas en las directrices de la tarea académica

\section{Patrón individual de contribuciones (PIC)}

Continuo: cuando el participante realiza sus contribuciones de manera permanente y distribuida en la mayoría de los días durante la última semana de actividad en el foro en línea

Discontinuo: cuando el participante realiza sus contribuciones de manera aislada y concentrada solo sobre un par de días durante la última semana de actividad en el foro en línea

\section{Índice individual de reciprocidad}

Valor umbral para el ejercicio adecuado de la IE: $\geq 0,63$

Cuando el participante establece relaciones comunicativas mutuas con la mayoría de los participantes del grupo
Indicadores grupales de la actividad en el foro Índice grupal de acceso (IGA)

Valor umbral para el ejercicio adecuado de la IE: $\geq 0,50$

Cuando el grupo en su conjunto accede al foro al menos en la mitad de los días que dura la actividad en el foro en línea

\section{Índice grupal de lecturas (IGL)}

Valor umbral para el ejercicio adecuado de la IE: $\geq 0,90$

Cuando el grupo en su conjunto lee prácticamente la totalidad de las contribuciones hechas en el foro en línea

\section{Índice grupal de contribuciones (IGC)}

Valor umbral para el ejercicio adecuado de la IE: $\geq 1,50$

Cuando el grupo en su conjunto realiza como mínimo un $50 \%$ más contribuciones que las exigidas en las directrices de la tarea académica

\section{Índice de densidad de la red (IDR) y sociograma}

Cuanto mayor sea el número de relaciones comunicativas que establecen los participantes entre sí, más se acercará el valor obtenido por el grupo al valor de densidad máxima $(1,00)$ y más cerca estará el grupo de conformar un sociograma en estrella.

\section{Índice grupal de reciprocidad}

Valor umbral para el ejercicio adecuado de la IE: $\geq 0,63$

Cuando el grupo en su conjunto presenta una alta proporción de pares de participantes que tienen relación comunicativa mutua

Nota. Adaptada de Engel, Coll y Bustos (2013).

La actividad de los participantes como fuente de información para promover la colaboración. Una analítica del aprendizaje basada en el modelo de Influencia Educativa Distribuida. César Coll, Anna Engel y Shamaly Niño. 
A los estudiantes del grupo 2 -G2- se les entregaba individualmente información relativa al contenido de las contribuciones atendiendo a las dimensiones de participación social, tarea académica y significados sobre los contenidos de aprendizaje, referida tanto al grupo en su conjunto como a cada participante individual. Para elaborar esta información dos jueces codificaban por separado las contribuciones en términos de las tres dimensiones implicadas en el ejercicio de la IE y comprobaban la fiabilidad de las codificaciones resultantes (Kappa de Cohen); en todos los casos los valores obtenidos fueron superiores al $90 \%$. En la Tabla 2 se describe el procedimiento utilizado para el cálculo de los indicadores individuales y grupales de gestión de la participación social, de la tarea y de los significados, de acuerdo con las definiciones presentadas más arriba; así mismo, se indican las categorías utilizadas para codificar las contribuciones o fragmentos de contribuciones en las tres dimensiones.

La actividad de los participantes como fuente de información para promover la colaboración. Una analítica del aprendizaje basada en el modelo de Influencia Educativa Distribuida. César Coll, Anna Engel y Shamaly Niño.

Página 12 de 36. 
Tabla 2. Información derivada del análisis de contenido proporcionada a los participantes del G2

Indicadores relativos al contenido de las contribuciones

Indicadores individuales de la actividad en el foro

Índice individual de la participación social (IIP)

Contribuciones o fragmentos de contribuciones de este tipo de gestión respecto al total de contribuciones o fragmentos de contribuciones que aporta el participante
Indicadores grupales de la actividad en el foro

\section{Índice grupal de la participación social (IGP)}

Contribuciones o fragmentos de contribuciones de este tipo de gestión respecto al total de contribuciones o fragmentos de contribuciones que aporta el grupo en su conjunto

i. Formulación o recordatorio de las reglas de participación en el foro

ii. Petición o exigencia de precisiones sobre las reglas de participación en el foro

iii. Formulación de precisiones sobre las reglas de participación a requerimiento de otros participantes

iv. Valoración de las reglas de participación.

v. Propuesta de revisión o reformulación de las reglas de participación en el foro

Índice individual de la tarea académica (IIT)

Contribuciones o fragmentos de contribuciones de este tipo de gestión respecto al total de contribuciones o fragmentos de contribuciones que aporta el participante

\section{Índice grupal de la tarea académica (IGT)}

Contribuciones o fragmentos de contribuciones de este tipo de gestión respecto al total de contribuciones o fragmentos de contribuciones que aporta el grupo en su conjunto

i. Formulación o recordatorio de las características o exigencias del glosario, su abordaje y su producto o resultado.

ii. Petición o exigencia de precisiones sobre las características del glosario, su abordaje y su producto o resultado.

iii. Formulación de precisiones sobre las características o exigencias del glosario, su abordaje y su producto o resultado. iv. Valoración de las características o exigencias del glosario, su abordaje y su producto o resultado.

v. Propuesta de revisión o reformulación de las características o exigencias del glosario, su abordaje y su producto.

vi. Anuncio de la realización de una acción relativa al glosario y/o elaboración de una o más definiciones.

vii. Cambios formales en las definiciones construidas (ortografía, puntuación)

Índice individual de los significados sobre los contenidos de aprendizaje (IIS)

Contribuciones o fragmentos de contribuciones de este tipo de gestión respecto al total de contribuciones o fragmentos de contribuciones que aporta el participante

\section{Índice grupal de los significados sobre los contenidos de aprendizaje (IGS) \\ Contribuciones o fragmentos de contribuciones de este tipo de gestión respecto al total de contribuciones o fragmentos de contribuciones que aporta el grupo en su conjunto}

i. Aportación a iniciativa propia de significados propios con un cierto grado de elaboración.

ii. Aportación a iniciativa propia de significados atribuidos a fuentes externas con un cierto grado de elaboración.

iii. Incorporación de significados mediante adjuntos documentales de autoría propia o ajena.

iv. Identificación de tópicos o temas de atención, indagación y discusión.

v. Recordatorio literal o casi literal de significados presentados previamente.

vi. Valoración favorable (manifestaciones de acuerdo y aceptación) de significados aportados previamente.

vii. Valoración crítica (manifestaciones de desacuerdo o discrepancia) de significados aportados previamente.

viii. Requerimiento a otros participantes para que aporten significados sobre un tópico.

ix. Respuesta a un requerimiento de otro participante.

x. Petición de precisiones a otro(s) participante(s) sobre los significados presentados previamente.

xi. Respuesta a una petición de precisiones.

xii. Identificación y/o corrección de errores o incomprensiones en los significados aportados previamente.

xiii. Expresión o manifestación de dudas o incomprensiones o de inseguridad respecto a uno o varios de los tópicos. xiv. Formulación de síntesis, resúmenes o recapitulaciones integrando los significados aportados previamente.

Nota. Adaptada de Coll, Bustos y Engel (2011).

\section{A los estudiantes del grupo 3 -G3- se les entregaba individualmente los dos tipos de información anteriores: la relativa al acceso, la participación y la conectividad, y la}

La actividad de los participantes como fuente de información para promover la colaboración. Una analítica del aprendizaje basada en el modelo de Influencia Educativa Distribuida. César Coll, Anna Engel y Shamaly Niño. 
relativa al contenido de sus contribuciones atendiendo a las tres dimensiones del ejercicio de la IE.

Por último, a los estudiantes del grupo 4-G4- se les proporcionaba de forma colectiva únicamente valoraciones generales sobre el cumplimiento de las directrices del profesor para la elaboración de los glosarios en el foro.

\section{Recogida y análisis de datos}

Para analizar las características del proceso colaborativo y su evolución en los cuatro grupos, hemos utilizado los registros de actividad que proporcionan las bases de datos de la plataforma Moodle y los registros de las contribuciones de los participantes. En el primer caso, el procedimiento utilizado ha consistido en calcular el conjunto de indicadores grupales e individuales de tipo estructural descritos en la Tabla 1 para los cuatro grupos en los siete foros. Los indicadores grupales informan del nivel de cumplimiento de las exigencias de acceso, participación y conectividad para el ejercicio de la IED en los cuatro grupos; así mismo, y bajo los mismos supuestos teóricos, los cambios en los valores de estos indicadores en los foros sucesivos informan de la evolución del proceso de colaboración en cada uno de los grupos. Por su parte, los indicadores individuales informan del nivel de cumplimiento de las exigencias de acceso, participación y conectividad para el ejercicio de la IE y, en consecuencia, de la implicación de los participantes en el desarrollo del proceso de colaboración. Así mismo, los cambios en los valores de estos indicadores en los foros sucesivos informan de la evolución de la implicación de los participantes individuales en el proceso de colaboración.

En el segundo caso, se han codificado las contribuciones de los participantes de los cuatro grupos en los siete foros atendiendo a las tres dimensiones implicadas en el ejercicio de la IE -participación, tarea, contenido- y se han calculado los indicadores grupales e individuales correspondientes de acuerdo con el procedimiento descrito en la Tabla 2. Los indicadores grupales informan del peso relativo de estas tres dimensiones en el ejercicio de la IED en cada uno de los grupos y de su evolución en el transcurso de los foros. Los indicadores individuales proporcionan información sobre el ejercicio de la IE de los participantes de cada grupo en las tres dimensiones y de su evolución en el transcurso de los foros.

\section{Resultados}

Presentamos, en primer lugar, los resultados correspondientes a los indicadores grupales e individuales de acceso, participación y conectividad en los cuatro grupos y su evolución en el transcurso de los siete foros; y, en segundo lugar, los correspondientes a los indicadores relativos al contenido de las contribuciones.

La actividad de los participantes como fuente de información para promover la colaboración. Una analítica del aprendizaje basada en el modelo de Influencia Educativa Distribuida. César Coll, Anna Engel y Shamaly Niño.

Página 14 de 36. 


\section{Indicadores grupales e individuales relativos al acceso, la participación y la conectividad y su evolución}

En la Tabla 3 se muestran los indicadores grupales de acceso, participación y conectividad con valores iguales o superiores (celdas sombreadas) y con valores inferiores (celdas sin sombrear) al umbral establecido como adecuado para el ejercicio de la IE en los siete foros. Como se puede ver, hay una diferencia neta en los cuatro grupos entre el F1, en el que los estudiantes no cuentan con información previa sobre su actividad, y los seis foros siguientes en los que sí cuentan con esta información. El hecho de que el F1 sea el foro en el que los cuatro grupos presentan un menor número de indicadores grupales con valores iguales o superiores al umbral establecido como idóneo para el ejercicio de la IE (solo 2 sobre 5 en G1 y G3, y 1 sobre 5 en G2 y G4) indica que, de acuerdo con el marco teórico adoptado, en términos generales en este foro no se cumplen las exigencias de acceso, participación y conectividad para que los participantes puedan ejercer la IE de manera apropiada. La diferencia se establece por lo tanto entre el F1 y el resto de los foros (F2 a F7) en los cuatro grupos. No hay en cambio diferencias claras entre los grupos en ningún foro en lo que concierne al número de indicadores con valores iguales o superiores al umbral establecido. Las diferencias entre los grupos, sin embargo, aparecen si nos fijamos en el número de foros en los que los cinco indicadores grupales presentan valores iguales o superiores al umbral establecido: 4/6 en G1, 2/6 en el G2, 3/6 en el G3 y 0/6 en el G4. No se observan en los grupos, por lo demás, patrones claros en los indicadores con valores por debajo del umbral establecido, excepto en lo que concierne al G4 en el que los valores del índice grupal de lectura (IGL) se encuentran por debajo del umbral en todos los foros.

La actividad de los participantes como fuente de información para promover la colaboración. Una analítica del aprendizaje basada en el modelo de Influencia Educativa Distribuida. César Coll, Anna Engel y Shamaly Niño.

Página 15 de 36. 
Tabla 3. Indicadores grupales de acceso, participación y conectividad de los cuatro grupos en los siete foros: valores iguales o superiores (celdas sombreadas) y valores inferiores (celdas sin sombrear) al umbral establecido como adecuado para el ejercicio de la IED

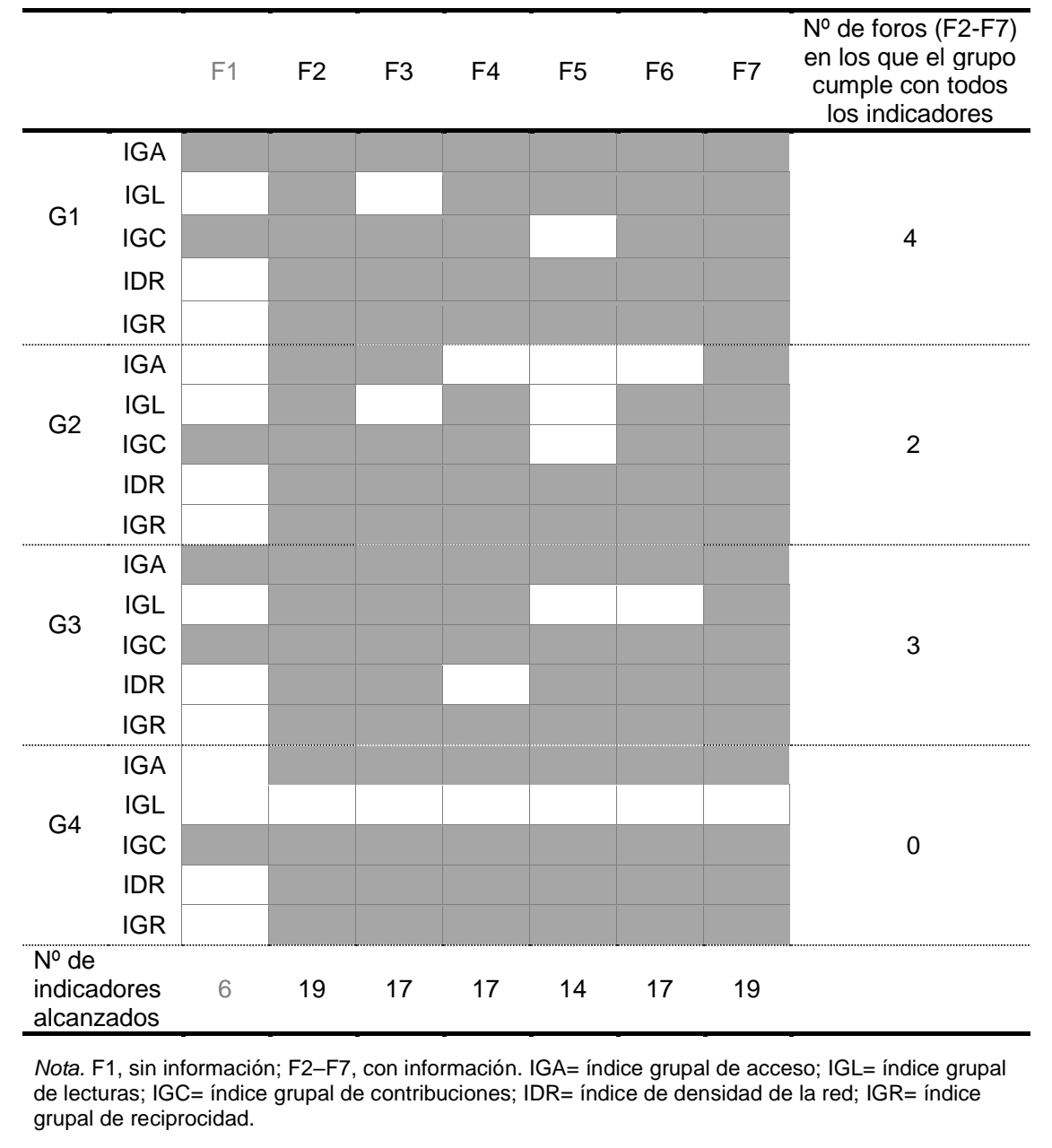

Pasando ahora a los indicadores individuales de acceso, participación y conectividad, en la Tabla 4 se muestra el número de estudiantes de los cuatro grupos que alcanzan valores iguales o superiores al establecido como adecuado para el ejercicio de la IE en al menos 5 de los 6 indicadores en los siete foros. Al igual que sucede con los indicadores grupales, de nuevo en los cuatro grupos la diferencia se produce entre el F1, en el que los estudiantes no cuentan con información previa sobre su actividad, y los seis foros siguientes, en los que sí que cuentan con algún tipo de información al respecto. Tampoco se observan diferencias claras entre los grupos en el número de estudiantes que alcanzan valores iguales o superiores al establecido como teóricamente adecuado para el ejercicio de IE ni en su evolución desde el F2 hasta el F7.

La actividad de los participantes como fuente de información para promover la colaboración. Una analítica del aprendizaje basada en el modelo de Influencia Educativa Distribuida. César Coll, Anna Engel y Shamaly Niño.

Página 16 de 36. 
Tabla 4. Número de estudiantes de los cuatro grupos con valores iguales o superiores al establecido como adecuado para el ejercicio de la IE en al menos 5 de los 6 indicadores individuales en los siete foros

\begin{tabular}{lccccccc}
\hline & $F 1$ & $F 2$ & $F 3$ & $F 4$ & $F 5$ & F6 & F7 \\
\hline G1 $(\mathrm{N}=9)$ & 2 & 8 & 6 & 8 & 5 & 8 & 7 \\
G2 $(\mathrm{N}=8)$ & 3 & 7 & 6 & 6 & 5 & 7 & 6 \\
G3 $(\mathrm{N}=8)$ & 2 & 8 & 7 & 6 & 3 & 6 & 5 \\
$\mathrm{G} 4(\mathrm{~N}=8)$ & 1 & 7 & 7 & 6 & 6 & 5 & 5 \\
\hline
\end{tabular}

Nota. F1, sin información; F2-F7, con información.

Las diferencias empiezan a aparecer, sin embargo, cuando observamos los valores de los diferentes índices individuales de acceso, participación y conectividad y su evolución por separado. Como muestra la Tabla 5, la información proporcionada a los participantes sobre su actividad entre el final del F1 y el inicio del F2 impacta de forma desigual en los valores de los indicadores en los foros siguientes. Así, el impacto es especialmente notorio y sostenido en el caso del índice individual de reciprocidad (IIR) en todos los grupos. En el extremo opuesto se sitúa el patrón individual de acceso (PIA), con un impacto prácticamente inexistente y valores relativamente estables entre el F2 y el F7. En el resto de los indicadores el impacto es en general menos homogéneo y menos sostenido, aunque con diferencias importantes entre ellos. Mención especial merecen el índice individual de contribuciones (IIC) y el índice individual de lecturas (IIL), dos indicadores cuyos valores aparecen estrechamente asociados en el marco teórico de referencia al nivel del ejercicio de la IE.

La actividad de los participantes como fuente de información para promover la colaboración. Una analítica del aprendizaje basada en el modelo de Influencia Educativa Distribuida. César Coll, Anna Engel y Shamaly Niño.

Página 17 de 36. 
Tabla 5. Número de estudiantes de los cuatro grupos con valores iguales o superiores al umbral establecido como adecuado para el ejercicio de la IE en los indicadores correspondientes en los siete foros

\begin{tabular}{|c|c|c|c|c|c|c|c|c|}
\hline & Indicadores & $\mathrm{F} 1$ & $\mathrm{~F} 2$ & F3 & $\mathrm{F} 4$ & F5 & F6 & F7 \\
\hline \multirow{6}{*}{$\mathrm{G} 1(\mathrm{~N}=9)$} & IIA & 4 & 7 & 9 & 8 & 6 & 8 & 9 \\
\hline & PIA & 9 & 9 & 9 & 9 & 9 & 9 & 8 \\
\hline & IIL & 4 & 7 & 5 & 8 & 8 & 8 & 7 \\
\hline & IIC & 5 & 9 & 7 & 9 & 4 & 8 & 8 \\
\hline & PIC & 5 & 9 & 6 & 8 & 7 & 8 & 5 \\
\hline & IIR & 3 & 9 & 7 & 8 & 7 & 9 & 9 \\
\hline \multirow{6}{*}{$\mathrm{G} 2(\mathrm{~N}=8)$} & IIA & 3 & 7 & 7 & 4 & 5 & 4 & 6 \\
\hline & PIA & 6 & 8 & 8 & 8 & 8 & 8 & 8 \\
\hline & IIL & 4 & 6 & 5 & 5 & 5 & 7 & 6 \\
\hline & IIC & 5 & 8 & 8 & 8 & 3 & 8 & 8 \\
\hline & PIC & 5 & 8 & 8 & 8 & 8 & 8 & 8 \\
\hline & IIR & 4 & 8 & 7 & 8 & 8 & 8 & 8 \\
\hline \multirow{6}{*}{ G3 $(N=8)$} & IIA & 5 & 8 & 7 & 6 & 3 & 6 & 5 \\
\hline & PIA & 7 & 8 & 8 & 6 & 7 & 7 & 8 \\
\hline & IIL & 2 & 7 & 7 & 6 & 6 & 5 & 6 \\
\hline & IIC & 5 & 8 & 8 & 7 & 4 & 7 & 6 \\
\hline & PIC & 5 & 8 & 7 & 6 & 5 & 4 & 5 \\
\hline & IIR & 1 & 8 & 8 & 7 & 6 & 7 & 6 \\
\hline \multirow{6}{*}{$\mathrm{G} 4(\mathrm{~N}=8)$} & $\| \mathrm{A}$ & 2 & 7 & 7 & 7 & 6 & 6 & 5 \\
\hline & PIA & 7 & 7 & 8 & 7 & 7 & 7 & 8 \\
\hline & IIL & 5 & 4 & 4 & 5 & 4 & 5 & 3 \\
\hline & IIC & 4 & 7 & 7 & 7 & 7 & 7 & 7 \\
\hline & PIC & 3 & 6 & 7 & 7 & 6 & 5 & 7 \\
\hline & IIR & 1 & 7 & 7 & 8 & 7 & 8 & 8 \\
\hline
\end{tabular}

Nota. F1, sin información; F2-F7, con información. IIA= índice individual de acceso; PIA= patrón individual de acceso; $\mathrm{IL}=$ índice individual de lecturas; $I \mathrm{IC}=$ índice individual de contribuciones; $\mathrm{PIC}=$ patrón individual de contribuciones; IIR= índice individual de reciprocidad.

En el caso del IIC el impacto de la información proporcionada a los estudiantes sobre su actividad en el $\mathrm{F} 1$ es elevado en los cuatro grupos y sus valores se mantienen relativamente estables entre el $\mathrm{F} 2$ y el F7, a excepción del F5 en el que el número de estudiantes con valores iguales o superiores al umbral establecido para este índice como apropiado para ejercicio de la IE disminuye sensiblemente en G1, G2 y G3. Una posible explicación de esta disminución es que el período durante el cual se desarrolla el F5 incluye un período vacacional, lo que puede haber motivado una menor implicación de algunos participantes en la tarea. Por otra parte, para interpretar adecuadamente el carácter en general sostenido del impacto en los valores de este índice en los cuatro grupos, hay que tener en cuenta que las instrucciones de desarrollo de los foros incluían la obligatoriedad de un cierto número de aportaciones de cada estudiante.

En lo que concierne al IIL, el impacto de la información proporcionada a los

La actividad de los participantes como fuente de información para promover la colaboración. Una analítica del aprendizaje basada en el modelo de Influencia Educativa Distribuida. César Coll, Anna Engel y Shamaly Niño.

Página 18 de 36. 
estudiantes sobre su actividad al término del F1 sólo se observa en los G1, G2 y G3, siendo además este impacto sostenido entre el F2 y el F7, aunque con fluctuaciones. Llama especialmente la atención, por otra parte, que en el G4 el número de estudiantes con valores iguales o superiores al umbral establecido para este índice como apropiado para ejercicio de la IE sea en todos los foros igual o inferior al del F1. Conviene recordar aquí que los estudiantes del G4 son los únicos que no reciben información detallada sobre su actividad al término de los foros, en contraposición a los estudiantes del G1, que reciben información sobre acceso, participación y conectividad, a los estudiantes del G2, que la reciben sobre el contenido de sus aportaciones, y a los del G3, que la reciben sobre ambos aspectos.

Con el fin de completar esta panorámica de los indicadores individuales de acceso, participación y conectividad, conviene aún aludir al hecho de que los estudiantes se distribuyen desigualmente en los cuatro grupos en lo que concierne al número de foros en los que presentan indicadores con valores iguales o superiores a los establecidos como umbrales para el ejercicio apropiado de la IE. En el G1, hay 3/9 estudiantes que presentan estos valores en al menos 5 de los 6 indicadores en los 6 foros (F2-F7), en el G2 5/8, en el G3 2/8 y en el G4 3/8. La Tabla 6 muestra esta distribución en detalle en los cuatro grupos.

La actividad de los participantes como fuente de información para promover la colaboración. Una analítica del aprendizaje basada en el modelo de Influencia Educativa Distribuida. César Coll, Anna Engel y Shamaly Niño.

Página 19 de 36. 
Tabla 6. Número de foros (F2 - F7) en los que los participantes de los cuatro grupos presentan indicadores individuales de acceso, participación y conectividad con valores iguales o superiores a los establecidos como umbrales para el ejercicio adecuado de la IE (mínimo: 0; máximo: 6)

\begin{tabular}{|c|c|c|c|c|c|c|c|c|c|}
\hline & & & \multicolumn{7}{|c|}{ Número de indicadores } \\
\hline & & & 0 & 1 & 2 & 3 & 4 & 5 & 6 \\
\hline \multirow{9}{*}{$\frac{-}{\stackrel{\circ}{3}}$} & \multirow{9}{*}{$\begin{array}{l}\sigma \\
\text { II } \\
\underline{2}\end{array}$} & E1 & - & 1 & - & 1 & 2 & 1 & 1 \\
\hline & & E2 & - & - & - & - & 1 & 1 & 4 \\
\hline & & E3 & - & - & - & - & - & 2 & 4 \\
\hline & & E4 & - & - & - & - & - & - & 6 \\
\hline & & E5 & - & - & - & 1 & 1 & 1 & 3 \\
\hline & & E6 & - & - & - & - & - & 2 & 4 \\
\hline & & E7 & - & - & - & - & - & 1 & 2 \\
\hline & & E8 & - & - & - & - & - & 3 & 2 \\
\hline & & E9 & - & - & - & - & - & 2 & 2 \\
\hline & \multirow{8}{*}{$\begin{array}{l}\widehat{\infty} \\
\underline{I}\end{array}$} & E1 & - & - & - & 1 & 1 & 1 & 3 \\
\hline & & E2 & - & - & - & - & - & 1 & 5 \\
\hline & & E3 & - & - & - & - & - & 4 & 2 \\
\hline & & E4 & - & - & - & - & - & - & 6 \\
\hline & & E5 & - & - & - & 1 & 5 & - & - \\
\hline & & E6 & - & - & - & - & - & - & 6 \\
\hline & & E7 & - & - & - & - & - & 3 & 3 \\
\hline & & E8 & - & - & - & - & - & 1 & 2 \\
\hline \multirow{8}{*}{$\begin{array}{l}\text { m } \\
\text { 울 } \\
\text { 눈 }\end{array}$} & \multirow{8}{*}{$\begin{array}{l}\widehat{\infty} \\
\underline{I}\end{array}$} & $\mathrm{E} 1$ & - & - & - & - & - & 1 & 5 \\
\hline & & E2 & - & - & - & - & 2 & 2 & 2 \\
\hline & & E3 & - & 1 & 1 & - & - & 1 & 3 \\
\hline & & E4 & 1 & - & 2 & 1 & - & 1 & 3 \\
\hline & & E5 & - & - & - & - & 1 & 2 & 3 \\
\hline & & E6 & - & - & - & 1 & - & 1 & 4 \\
\hline & & E7 & - & - & 1 & 1 & 1 & 1 & 2 \\
\hline & & E8 & - & - & - & - & - & 2 & 4 \\
\hline \multirow{8}{*}{\multicolumn{2}{|c|}{ 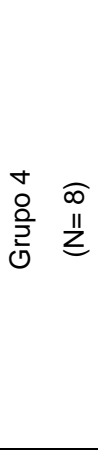 }} & $\mathrm{E} 1$ & - & - & - & - & - & 4 & 2 \\
\hline & & E2 & - & - & 1 & 3 & 1 & - & 1 \\
\hline & & E3 & - & - & - & 1 & 1 & 3 & 0 \\
\hline & & E4 & - & - & - & - & 1 & 2 & 3 \\
\hline & & E5 & - & - & 1 & - & 1 & 1 & 3 \\
\hline & & E6 & - & - & - & & 1 & 4 & 1 \\
\hline & & E7 & - & - & - & - & - & 3 & 3 \\
\hline & & E8 & - & - & - & - & - & 2 & 4 \\
\hline
\end{tabular}

La actividad de los participantes como fuente de información para promover la colaboración. Una analítica del aprendizaje basada en el modelo de Influencia Educativa Distribuida. César Coll, Anna Engel y Shamaly Niño.

Página 20 de 36. 


\section{Indicadores grupales e individuales relativos al contenido de las contribuciones y su evolución}

La Tabla 7 muestra el número total de contribuciones o fragmentos de contribuciones codificadas en cada una de las tres dimensiones, su equivalente en términos porcentuales y los correspondientes indicadores grupales en el conjunto de los foros F2-F7. En los cuatro grupos las contribuciones codificadas como gestión de los significados sobre los contenidos de aprendizaje son las más numerosas, seguidas a una distancia considerable por las de tarea y, en último lugar, las de participación. Más allá de esta similitud en el patrón de distribución de las contribuciones entre las tres dimensiones se observa, sin embargo, algunas singularidades. Así, en el caso del G3 llama la atención el porcentaje relativamente elevado de contribuciones sobre participación en comparación con los de los otros grupos $(12,11 \%$ del total de contribuciones, frente $2,57 \%$ en el $\mathrm{G} 1,2,81 \%$ en el $\mathrm{G} 2$ y $4,99 \%$ en el G4) y, en contrapartida, el porcentaje relativamente bajo -pese a seguir siendo la dimensión más frecuente- de contribuciones sobre los contenidos $(54,40 \%$ del total, frente al $70,29 \%$ en el G1, 60,23\% en el G2 y $61,38 \%$ en el G4). Así mismo, destaca que el G1, con el porcentaje más elevado de contribuciones sobre los contenidos, sea al mismo tiempo el que presenta porcentajes más bajos de contribuciones sobre la tarea (27,14\% del total, frente a $36,95 \%$ en el G2, 33,49\% en el G3 y $33,63 \%$ en el G4) y la participación.

\begin{tabular}{|c|c|c|c|c|c|}
\hline & & Participación & Tarea & Significados & Totales \\
\hline \multirow{3}{*}{ G1 } & $\mathrm{N}$ & 52 & 549 & 1,422 & 2,023 \\
\hline & $\%$ & $2,57 \%$ & $27,14 \%$ & $70,29 \%$ & $100 \%$ \\
\hline & IG & 0,026 & 0,271 & 0,703 & - \\
\hline \multirow{3}{*}{ G2 } & $\mathrm{N}$ & 72 & 946 & 1,542 & 2,560 \\
\hline & $\%$ & $2,81 \%$ & $36,95 \%$ & $60,23 \%$ & $100 \%$ \\
\hline & IG & 0,028 & 0,370 & 0,602 & - \\
\hline \multirow{3}{*}{ G3 } & $N$ & 259 & 716 & 1,163 & 2,138 \\
\hline & $\%$ & $12,11 \%$ & $33,49 \%$ & $54,40 \%$ & $100 \%$ \\
\hline & IG & 0,121 & 0,335 & 0,544 & - \\
\hline \multirow{3}{*}{ G4 } & $\mathrm{N}$ & 125 & 842 & 1,537 & 2,504 \\
\hline & $\%$ & $4,99 \%$ & $33,63 \%$ & $61,38 \%$ & $100 \%$ \\
\hline & IG & 0,050 & 0,336 & 0,614 & - \\
\hline
\end{tabular}

Como puede verse en la Tabla 8 estas singularidades de los grupos G1 y G3 están presentes en prácticamente todos los foros a partir del F2. Así, en comparación con los de los otros grupos, los IGP del G3 son los más altos y, en contrapartida, los IGS son los más bajos con una sola excepción (F6). Del mismo modo, los IGS del G1 son

La actividad de los participantes como fuente de información para promover la colaboración. Una analítica del aprendizaje basada en el modelo de Influencia Educativa Distribuida. César Coll, Anna Engel y Shamaly Niño.

Página 21 de 36. 
los más elevados en cuatro de los seis foros (las excepciones son el F5 y el F7), mientras que los IGP y los IGT tienden a situarse entre los más bajos. Algo similar sucede en el grupo G2, con valores intermedios en los IGS, altos en los IGT y bajos en los IGP en la mayoría de los foros; y en el grupo G4, con valores intermedios en los tres indicadores en la mayoría de los foros.

\begin{tabular}{|c|c|c|c|c|c|c|c|c|c|}
\hline & & F1 & $\mathrm{F} 2$ & F3 & $\mathrm{F} 4$ & F5 & F6 & F7 & $\begin{array}{r}\text { F2 - F7 } \\
\text { Media }\end{array}$ \\
\hline \multirow{3}{*}{$\mathrm{G} 1$} & IGP & 0,075 & 0,025 & 0,022 & 0,028 & 0,030 & 0,026 & 0,025 & 0,026 \\
\hline & IGT & 0,281 & 0,248 & 0,299 & 0,186 & 0,317 & 0,256 & 0,335 & 0,271 \\
\hline & IGS & 0,643 & 0,727 & 0,679 & 0,786 & 0,653 & 0,718 & 0,641 & 0,703 \\
\hline \multirow{3}{*}{ G2 } & IGP & 0,178 & 0,027 & 0,025 & 0,047 & 0,029 & 0,023 & 0,020 & 0,028 \\
\hline & IGT & 0,324 & 0,307 & 0,347 & 0,336 & 0,366 & 0,486 & 0,356 & 0,370 \\
\hline & IGS & 0,498 & 0,665 & 0,627 & 0,617 & 0,606 & 0,490 & 0,624 & 0,602 \\
\hline \multirow{3}{*}{ G3 } & IGP & 0,092 & 0,186 & 0,089 & 0,091 & 0,166 & 0,046 & 0,056 & 0,121 \\
\hline & IGT & 0,397 & 0,333 & 0,403 & 0,315 & 0,296 & 0,303 & 0,349 & 0,335 \\
\hline & IGS & 0,510 & 0,481 & 0,508 & 0,594 & 0,538 & 0,651 & 0,595 & 0,544 \\
\hline \multirow{3}{*}{ G4 } & IGP & 0,075 & 0,093 & 0,030 & 0,043 & 0,063 & 0,045 & 0,017 & 0,050 \\
\hline & IGT & 0,310 & 0,428 & 0,350 & 0,337 & 0,258 & 0,332 & 0,324 & 0,336 \\
\hline & IGS & 0,615 & 0,479 & 0,620 & 0,620 & 0,679 & 0,622 & 0,659 & 0,614 \\
\hline
\end{tabular}

Nota. F1, sin información; F2-F7, con información.

De acuerdo con el marco teórico adoptado, estos resultados indican que en los cuatro grupos el ejercicio de la IE se sitúa en primer lugar en la dimensión de la gestión de los significados sobre los contenidos; en segundo lugar, y con una frecuencia considerablemente menor, en la gestión de la tarea; y, por último, y con una frecuencia ya muy baja, en la gestión de la participación social. Junto a este patrón compartido, sin embargo, la lectura conjunta de las tablas 5 y 6 invita a pensar que las singularidades detectadas en los pesos relativos de las tres dimensiones son el reflejo de unas dinámicas grupales que, una vez establecidas, tienden a mantenerse. El análisis de las contribuciones individuales de los participantes, y más concretamente de los índices individuales de participación (IIP), tarea (IIT) y significados (IIS) en los cuatro grupos, así como de su evolución entre el F2 y el F7, proporciona una primera clave para describir y entender estas dinámicas.

La actividad de los participantes como fuente de información para promover la colaboración. Una analítica del aprendizaje basada en el modelo de Influencia Educativa Distribuida. César Coll, Anna Engel y Shamaly Niño.

Página 22 de 36. 
RED. Revista de Educación a Distancia, 53, Artíc. 2. 31-Marzo-2017 http://www.um.es/ead/red/53

Tabla 9. Contribuciones o fragmentos de contribuciones de los miembros de los grupos codificados como gestión de la participación $(P)$, de la tarea $(T)$ y de los significados $(S)$ en el conjunto de los foros F2-F7 e indicadores individuales en las tres dimensiones (IIP, IIT, IIS)

\begin{tabular}{|c|c|c|c|c|c|c|c|c|c|c|c|c|}
\hline & & & \multicolumn{10}{|c|}{ Fragmentos de contribuciones de los estudiantes en cada grupo } \\
\hline \multirow{8}{*}{ G1 } & \multirow{3}{*}{$\mathbf{P}$} & & E1 & E2 & E3 & E4 & E5 & E6 & E7 & E8 & E9 & Total \\
\hline & & $\mathbf{n}$ & 3 & 5 & 12 & 4 & 3 & 10 & 8 & 7 & 0 & 52 \\
\hline & & IIP & 0,018 & 0,018 & 0,025 & 0,023 & 0,016 & 0,034 & 0,045 & 0,040 & 0,000 & $0,026\left(^{*}\right)$ \\
\hline & \multirow{2}{*}{$\mathbf{T}$} & $\mathrm{n}$ & 35 & 69 & 139 & 41 & 48 & 90 & 48 & 52 & 27 & 549 \\
\hline & & IIT & 0,215 & 0,253 & 0,294 & 0,234 & 0,262 & 0,306 & 0,268 & 0,299 & 0,245 & $0,271\left(^{*}\right)$ \\
\hline & \multirow{2}{*}{$\mathbf{S}$} & $\mathrm{n}$ & 125 & 199 & 321 & 130 & 132 & 194 & 123 & 115 & 83 & 1422 \\
\hline & & IIS & 0,767 & 0,729 & 0,680 & 0,743 & 0,721 & 0,660 & 0,687 & 0,661 & 0,755 & $0,703\left({ }^{*}\right)$ \\
\hline & Total & $\mathbf{N}$ & 163 & 273 & 472 & 175 & 183 & 294 & 179 & 174 & 110 & 2023 \\
\hline \multirow{8}{*}{ G2 } & \multirow{3}{*}{$\mathbf{P}$} & & E1 & E2 & E3 & E4 & E5 & E6 & E7 & E8 & Total & \\
\hline & & n & 3 & 6 & 15 & 14 & 4 & 11 & 12 & 7 & 72 & \\
\hline & & IIP & 0,016 & 0,022 & 0,027 & 0,038 & 0,011 & 0,031 & 0,051 & 0,030 & $0,028\left(^{*}\right)$ & \\
\hline & & $n$ & 83 & 93 & 195 & 151 & 139 & 118 & 88 & 79 & 946 & \\
\hline & & IIT & 0,444 & 0,336 & 0,349 & 0,410 & 0,402 & 0,335 & 0,374 & 0,335 & $0,370\left(^{*}\right)$ & \\
\hline & \multirow{2}{*}{ S } & $\mathrm{n}$ & 101 & 178 & 349 & 203 & 203 & 223 & 135 & 150 & 1542 & \\
\hline & & IIS & 0,540 & 0,643 & 0,624 & 0,552 & 0,587 & 0,634 & 0,574 & 0,636 & $0,602\left(^{*}\right)$ & \\
\hline & Total & $\mathbf{N}$ & 187 & 277 & 559 & 368 & 346 & 352 & 235 & 236 & 2560 & \\
\hline \multirow{8}{*}{ G3 } & \multirow{3}{*}{$\mathbf{P}$} & & E1 & E2 & E3 & E4 & E5 & E6 & E7 & E8 & Total & \\
\hline & & n & 76 & 15 & 33 & 9 & 37 & 19 & 46 & 24 & 259 & \\
\hline & & IIP & 0,132 & 0,064 & 0,205 & 0,071 & 0,111 & 0,134 & 0,127 & 0,118 & $0,121\left(^{*}\right)$ & \\
\hline & & $\mathbf{n}$ & 204 & 73 & 49 & 41 & 103 & 65 & 111 & 70 & 716 & \\
\hline & & IIT & 0,355 & 0,312 & 0,304 & 0,323 & 0,308 & 0,458 & 0,307 & 0,343 & $0,335\left(^{*}\right)$ & \\
\hline & \multirow{2}{*}{$S$} & $n$ & 294 & 146 & 79 & 77 & 194 & 58 & 205 & 110 & 1163 & \\
\hline & & IIS & 0,512 & 0,624 & 0,491 & 0,606 & 0,581 & 0,408 & 0,566 & 0,534 & $0,544\left(^{*}\right)$ & \\
\hline & Total & $\mathbf{N}$ & 574 & 234 & 161 & 127 & 334 & 142 & 362 & 204 & 2138 & \\
\hline \multirow{3}{*}{ G4 } & \multirow{3}{*}{$\mathbf{P}$} & & E1 & E2 & E3 & E4 & E5 & E6 & E7 & E8 & Total & \\
\hline & & $n$ & 30 & 7 & 4 & 5 & 12 & 43 & 10 & 14 & 125 & \\
\hline & & IP & 0044 & ( 030 & (203 & ( & 0050 & 0112 & ( & (205? & 0.050 (*) $^{*}$ & \\
\hline
\end{tabular}

La actividad de los participantes como fuente de información para promover la colaboración. Una analítica del aprendizaje basada en el modelo de Influencia Educativa Distribuida. César Coll, Anna Engel y Shamaly Niño.

Página 23 de 36. 


$\begin{array}{cccccccccccc} & \mathbf{n} & 198 & 56 & 49 & 54 & 69 & 178 & 177 & 61 & \mathbf{8 4 2} \\ \mathbf{T} & \mathbf{I I T} & 0,293 & 0,311 & 0,280 & 0,276 & 0,337 & 0,468 & 0,419 & 0,226 & \mathbf{0 , 3 3 6}\left({ }^{*}\right) \\ & \mathbf{n} & 448 & 117 & 122 & 137 & 124 & 159 & 235 & 195 & \mathbf{1 5 3 7} \\ \mathbf{S} & \mathbf{I I S} & 0,663 & 0,650 & 0,697 & 0,699 & 0,605 & 0,418 & 0,557 & 0,722 & \mathbf{0 , 6 1 4}\left({ }^{*}\right) \\ & & & & & & & & & & \\ \text { Total } & \mathbf{N} & \mathbf{6 7 6} & \mathbf{1 8 0} & \mathbf{1 7 5} & \mathbf{1 9 6} & \mathbf{2 0 5} & \mathbf{3 8 0} & \mathbf{4 2 2} & \mathbf{2 7 0} & \mathbf{2 5 0 4}\end{array}$

Nota. $\left({ }^{*}\right)$ Índices grupales de gestión de la participación social (IGP), de la tarea (IGT) y de los significados (IGS). Casillas sombreadas= índices individuales con valores iguales o superiores a los valores de los índices grupales correspondientes.

La lectura de la Tabla 9, en la que se recogen estos índices, muestra la presencia de dos patrones o modalidades en el ejercicio de la IE en los foros. Por una parte, están los estudiantes cuyos valores en los índices de participación, tarea o ambos se sitúan por encima de los valores de los índices grupales correspondientes (casillas sombreadas de las filas $\mathrm{P}$ y $\mathrm{T}$ de los cuatro grupos). Por otra parte, están aquellos cuyos valores en el índice de gestión de significados sobre los contenidos de aprendizaje se sitúan por encima de los valores del índice grupal correspondiente (casillas sombreadas de las filas $S$ de los cuatro grupos). Solo 4 de los 33 estudiantes participantes en los foros presentan una variante ligeramente distinta de la segunda modalidad que consiste en presentar valores en los índices de significados y de participación por encima de los valores de los índices grupales correspondientes (E6 y E8 del G2; E7 del G3; E8 del G4). Se detecta así una cierta especialización en el ejercicio de la IE, de manera que, mientras unos estudiantes ejercen la IE de forma prioritaria en las dimensiones de participación y tarea, otros lo hacen en la dimensión de significados. Ambas modalidades de ejercicio de la IE aparecen, por lo demás, de forma equilibrada en los cuatro grupos ( 4 y 5 estudiantes respectivamente en el G1; 6 y 4 en el G2; 5 y 4 en el G3; 4 y 5 en el G4), no apreciándose diferencias importantes entre ellos.

Se verifica, por lo tanto, que en los cuatro grupos sus miembros asumen conjuntamente el ejercicio de la IE en las tres dimensiones postuladas como esenciales para un desarrollo adecuado de los procesos de aprendizaje colaborativo. Sería un error, sin embargo, inferir a partir de estos datos que la dinámica del proceso de colaboración es similar en los cuatro grupos. Para captar cabalmente esta dinámica es necesario aún completar y modular la información proporcionada por los índices individuales de participación, tarea y significados con dos informaciones complementarias. La primera es el peso de la dimensión a la que refiere el índice individual que estemos considerando en el conjunto de contribuciones del grupo. Así, por mencionar solo un ejemplo, es razonable suponer que el impacto de los índices individuales de participación en la dinámica del G1 (ver Tabla 7), en el que las contribuciones de los estudiantes en esta dimensión constituyen el 2,57\% del total de las contribuciones, será sensiblemente menor que el impacto en esa dinámica de los

La actividad de los participantes como fuente de información para promover la colaboración. Una analítica del aprendizaje basada en el modelo de Influencia Educativa Distribuida. César Coll, Anna Engel y Shamaly Niño.

Página 24 de 36. 
índices individuales de gestión de los significados, que en el mismo grupo constituyen el $70,29 \%$ del total de las contribuciones. La segunda información complementaria tiene que ver con cómo se sitúa el número de contribuciones de un miembro del grupo en la dimensión a la que refiere el índice individual que estemos considerando respecto a la media de contribuciones de los miembros del grupo en esa misma dimensión. Así, por mencionar de nuevo solo un ejemplo, es razonable suponer que el impacto del índice individual de gestión de significados del E1 en el G3 (ver Tabla 9), cuyo valor $(0,512)$ se encuentra por debajo del valor del índice grupal correspondiente $(0,544)$ pero cuyo número de contribuciones en esta dimensión (294) está muy por encima de la media de contribuciones de los miembros del grupo en esta dimensión (145), será menor que impacto del índice individual de gestión de significados del E4 en ese mismo grupo que, pese a tener un valor $(0,606)$ superior al del índice grupal en esa dimensión $(0,544)$, está basado en un número de contribuciones $(77)$ muy inferior a la media de contribuciones del grupo (145).

La toma en consideración de estas dos informaciones complementa la visión aportada por la identificación de las modalidades de ejercicio de la IE de los participantes mediante una valoración del impacto de sus aportaciones en la dinámica del proceso colaborativo. A este fin, hemos considerado que la aportación de un participante al ejercicio de la IE en una dimensión determinada tiene un impacto elevado sobre la dinámica del proceso colaborativo cuando el número de sus contribuciones en la dimensión en cuestión se sitúa en la mediana o por encima de la mediana de las contribuciones de los miembros del grupo en esa dimensión. La Tabla 10 muestra, para los cuatro grupos, los foros en los que las contribuciones de los estudiantes cumplen este criterio en las dimensiones de tarea y significados. La dimensión de gestión de la participación social no ha sido incluida debido a que, con la única excepción de los foros F2 y F5 en el grupo 3, el número de contribuciones codificadas en esta dimensión se sitúa en todos los casos por debajo del $10 \%$ del total de contribuciones.

La actividad de los participantes como fuente de información para promover la colaboración. Una analítica del aprendizaje basada en el modelo de Influencia Educativa Distribuida. César Coll, Anna Engel y Shamaly Niño.

Página 25 de 36. 
Tabla 10. Foros (F2-F7) en los que el número de contribuciones de los estudiantes en las dimensiones de tarea (T) y significados (S) se sitúa en la mediana o por encima de la mediana de las contribuciones de los miembros del grupo de referencia

\begin{tabular}{|c|c|c|c|c|c|c|c|c|c|c|}
\hline \multicolumn{11}{|c|}{ Número de foros de los estudiantes en cada grupo } \\
\hline \multirow{5}{*}{ G1 } & & $\overline{\mathrm{E1}}$ & $\overline{\mathrm{E2}}$ & $\overline{E 3}$ & $\overline{\mathrm{E} 4}$ & $\overline{E 5}$ & $\overline{E 6}$ & $\overline{E 7}$ & $\overline{\mathrm{E8}}$ & E9 \\
\hline & & 1 & 5 & 6 & 2 & 3 & 6 & 4 & 4 & 1 \\
\hline & $\mathbf{T}$ & F3 & $\begin{array}{l}\text { F2-F4-F5- } \\
\text { F6-F7 }\end{array}$ & $\begin{array}{l}\text { F2-F3-F4- } \\
\text { F5-F6-F7 }\end{array}$ & F2-F3 & F2-F3-F5 & $\begin{array}{l}\text { F2-F3-F4- } \\
\text { F5-F6-F7 }\end{array}$ & $\begin{array}{c}\text { F4-F5-F6- } \\
\text { F7 }\end{array}$ & $\begin{array}{c}\text { F4-F5-F6- } \\
\text { F7 }\end{array}$ & $\mathrm{F} 7$ \\
\hline & & 2 & 6 & 6 & 5 & 3 & 6 & 2 & 1 & \\
\hline & $\mathbf{S}$ & F4-F7 & $\begin{array}{l}\text { F2-F3-F4- } \\
\text { F5-F6-F7 }\end{array}$ & $\begin{array}{l}\text { F2-F3-F4- } \\
\text { F5-F6-F7 }\end{array}$ & $\begin{array}{l}\text { F2-F3-F4- } \\
\text { F5-F6 }\end{array}$ & F2-F3-F5 & $\begin{array}{l}\text { F2-F3-F4- } \\
\text { F5-F6-F7 }\end{array}$ & F5-F6 & F7 & - \\
\hline \multirow{5}{*}{ G2 } & & E1 & E2 & E3 & E4 & E5 & E6 & E7 & E8 & \\
\hline & & 1 & - & 6 & 6 & 4 & 5 & 1 & 1 & \\
\hline & $\mathbf{T}$ & F6 & - & $\begin{array}{l}\text { F2-F3-F4- } \\
\text { F5-F6-F7 }\end{array}$ & $\begin{array}{l}\text { F2-F3-F4- } \\
\text { F5-F6-F7 }\end{array}$ & $\begin{array}{l}\text { F2-F3- } \\
\text { F4-F7 }\end{array}$ & $\begin{array}{l}\text { F2-F3-F4- } \\
\text { F5-F6 }\end{array}$ & F5 & F7 & \\
\hline & & - & 3 & 6 & 4 & 5 & 4 & - & 2 & \\
\hline & $S$ & - & F2-F5-F6 & $\begin{array}{l}\text { F2-F3-F4- } \\
\text { F5-F6-F7 }\end{array}$ & $\begin{array}{c}\text { F3-F4-F5- } \\
\text { F6 }\end{array}$ & $\begin{array}{l}\text { F2-F3- } \\
\text { F4-F5-F7 }\end{array}$ & $\begin{array}{l}\text { F2-F3-F6- } \\
\text { F7 }\end{array}$ & - & F4-F7 & \\
\hline \multirow{5}{*}{ G3 } & & E1 & E2 & E3 & E4 & E5 & E6 & E7 & E8 & \\
\hline & & 6 & 3 & 2 & - & 4 & 1 & 5 & 3 & \\
\hline & $\mathbf{T}$ & $\begin{array}{l}\text { F2-F3-F4- } \\
\text { F5-F6-F7 }\end{array}$ & F3-F6-F7 & F4-F6 & - & $\begin{array}{l}\text { F2-F3- } \\
\text { F4-F5 }\end{array}$ & F2 & $\begin{array}{l}\text { F2-F3-F4- } \\
\text { F5-F7 }\end{array}$ & F5-F6-F7 & \\
\hline & & 6 & 5 & 1 & 1 & 4 & - & 5 & 3 & \\
\hline & $S$ & $\begin{array}{l}\text { F2-F3-F4- } \\
\text { F5-F6-F7 }\end{array}$ & $\begin{array}{c}\text { F3-F4-F5- } \\
\text { F6-F7 }\end{array}$ & F6 & F2 & $\begin{array}{l}\text { F2-F3- } \\
\text { F4-F5 }\end{array}$ & - & $\begin{array}{c}\text { F2-F3-F5- } \\
\text { F6-F7 }\end{array}$ & F4-F5-F7 & \\
\hline \multirow{5}{*}{ G4 } & & E1 & E2 & E3 & E4 & E5 & E6 & E7 & E8 & \\
\hline & & 6 & 1 & 1 & 2 & 2 & 5 & 6 & 1 & \\
\hline & $\mathbf{T}$ & $\begin{array}{l}\text { F2-F3-F4- } \\
\text { F5-F6-F7 }\end{array}$ & F2 & F5 & F6-F7 & F3-F6 & $\begin{array}{l}\text { F2-F3-F4- } \\
\text { F5-F7 }\end{array}$ & $\begin{array}{l}\text { F2-F3-F4- } \\
\text { F5-F6-F7 }\end{array}$ & F4 & \\
\hline & & 6 & - & 1 & 3 & 1 & 3 & 6 & 4 & \\
\hline & $S$ & $\begin{array}{l}\text { F2-F3-F4- } \\
\text { F5-F6-F7 }\end{array}$ & - & $\mathrm{F} 4$ & F3-F5-F7 & F6 & F2-F3-F7 & $\begin{array}{l}\text { F2-F3-F4- } \\
\text { F5-F6-F7 }\end{array}$ & $\begin{array}{l}\text { F2-F4-F5- } \\
\text { F6 }\end{array}$ & \\
\hline
\end{tabular}

Nota. No se han incluido los datos correspondientes a la dimensión de participación social debido a que -con la única excepción de los foros F2 y F5 en el grupo 3- el número de contribuciones en esta dimensión se sitúa por debajo del 10\% del total de contribuciones. En los dos foros mencionados -F2 y F5- los sujetos con un número de contribuciones codificadas como participación social en la mediana o por encima de la mediana son E1, E3, E5 y E7.

En el G1 hay tres participantes (E2, E3 y E6) que cumplen el criterio mencionado en ambas dimensiones en prácticamente todos los foros. En el extremo opuesto encontramos dos participantes (E9 y E1) que lo cumplen en un número muy reducido

La actividad de los participantes como fuente de información para promover la colaboración. Una analítica del aprendizaje basada en el modelo de Influencia Educativa Distribuida. César Coll, Anna Engel y Shamaly Niño.

Página 26 de 36. 
de foros en las dos dimensiones. Los otros cuatro participantes cumplen el criterio en un número intermedio de foros, algunos en mayor medida en la dimensión de tarea (E8 y E7), y algunos en la de significados (E4 y E5). La lectura por dimensiones indica que en la dimensión de tarea cinco participantes (E2, E3, E6, E7 y E8) cumplen el criterio en más de la mitad de los foros, mientras que solo dos (E9 y E1) lo hacen en uno y los dos restantes (E5 y E4) que lo cumplen en un número intermedio de foros. Algo similar sucede en la dimensión de significados, con cuatro participantes (E2, E3, E4 y E6) que lo cumplen en más de la mitad de los foros, dos (E9 y E8) que no lo cumplen en prácticamente ninguno, y los tres restantes (E5, E7 y E1) que lo cumplen en un número intermedio de foros. Desde el punto de vista de la IE, y más concretamente del impacto de la IE ejercida por los participantes en la dinámica del proceso colaborativo, estos resultados permiten identificar cuatro tipos de participantes: los que tienen un impacto elevado en la dinámica del proceso colaborativo en lo que concierne al ejercicio de la IE en la dimensión de tarea y de significados en todos los foros (E2, E3 y E6); los que tienen un impacto elevado en ambas dimensiones en muy pocos foros (E9 y E1); los que tienen un impacto elevado en un número intermedio de foros y este impacto se concentra mayoritariamente en la dimensión de tarea (E8 y E7); y, por último, los que tienen igualmente un impacto elevado en un número intermedio de foros, pero este impacto se concentra mayoritariamente en la dimensión de significados (E4 y E5).

El G2 presenta un panorama sensiblemente distinto al que acabamos de describir. Como se puede ver en la Tabla 10, en este grupo no hay prácticamente participantes de los dos tipos intermedios identificados en el G1: o bien cumplen el criterio en todos o la mayoría de los foros (E3, E4, E5, E6), o bien lo cumplen en muy pocos o en ninguno (E1, E7 y E8). La excepción es el E2, que lo cumple en la mitad de los foros, pero solo en la dimensión de significados. En otras palabras, los participantes cuyas aportaciones tienen un mayor impacto en la dinámica del proceso colaborativo tienden a ser los mismos en todos los foros, tanto en lo que concierne a la dimensión de tarea como a la de significados.

En el G3 encontramos de nuevo, como en el G1, una mayor dispersión de los participantes en lo que concierne al impacto de sus contribuciones en las dos dimensiones, aunque también aparecen algunas singularidades. En primer lugar, solo dos participantes (E1 y E7) cumplen el criterio de hacer un número de contribuciones que se sitúa por encima de la mediana en ambas dimensiones en prácticamente todos los foros. Hay, sin embargo, otros tres (E2, E5 y E8) que lo cumplen en al menos la mitad de los foros. Y solo tres (E6, E4 y E3) que lo cumplen en un número muy pequeño de foros. En suma, cinco de los ocho participantes hacen aportaciones de tarea y de significados en la mitad o más de la mitad de los foros con un impacto elevado en la dinámica del proceso colaborativo.

El G4 presenta también características singulares. Por una parte, volvemos a

La actividad de los participantes como fuente de información para promover la colaboración. Una analítica del aprendizaje basada en el modelo de Influencia Educativa Distribuida. César Coll, Anna Engel y Shamaly Niño.

Página 27 de 36. 
encontrar los tipos de participantes ya identificados en los grupos anteriores: los que cumplen el criterio en ambas dimensiones en la totalidad (E1 y E7) o la mayoría (E6) de los foros; los que lo cumplen en un número muy reducido de foros (E2, E3 y E5); y los que lo cumplen en un número intermedio de foros y de forma mayoritaria en una dimensión (E4 y E8). Por otra parte, la lectura de ambas dimensiones por separado sugiere una cierta especialización en el ejercicio de la IE, en el sentido de que hay participantes que tienden a centrar sus intervenciones en una dimensión en mayor medida que en la otra. Esto se manifiesta con claridad, por ejemplo, en el caso de E8, con cuatro foros en los que se cumple el criterio en la dimensión de significados frente a uno solo en el que se cumple en la dimensión de tarea. En la misma dirección, pero en sentido contrario, encontramos a E6, con cinco foros en los que se cumple el criterio en la dimensión de tarea y tres foros en la de significados.

\section{Discusión y conclusiones}

Las analíticas del aprendizaje se han convertido en un importante campo de estudio para mejorar los procesos de aprendizaje mediante el diseño de herramientas que ayuden a los diferentes usuarios a tener una mejor comprensión de estos procesos (Siemens y Long, 2011). Algunos estudios se han dirigido a analizar cómo una analítica del aprendizaje puede proporcionar a los profesores una visión profunda del proceso de aprendizaje (Fidalgo-Blanco et al., 2015; Haya et al., 2015; RodríguezTriana et al., 2015), mientras que otros han puesto el foco en el diseño de algoritmos que automatizan la minería de datos (Romero et al., 2007), o en la elaboración de propuestas visuales para presentar los resultados de los análisis (Mazza y Dimitrova, 2007; Valentín y Burgos, 2015).

En este contexto, y atendiendo a la importancia que tiene actualmente "aprender a colaborar" (Wilson y Scalise, 2015; Jenkins et al., 2006; Binkley et al., 2012) y a la necesidad de diseñar herramientas que ayuden a los participantes a optimizar los procesos que permiten aprender unos de otros (Siemens y Long, 2011), en este artículo hemos presentado, por un lado, las líneas directrices de una analítica del aprendizaje inspirada en el modelo teórico de la Influencia Educativa Distribuida (Bustos, 2011; Coll et al., 2015; Coll y Engel, 2014), y por otro, su implementación en un conjunto de foros de conversación en línea. Recordemos aún que esta implementación ha estado presidida por tres objetivos: discernir si la comunicación a los participantes de la información resultante de aplicar la analítica tiene un impacto sobre el proceso colaborativo que están llevando a cabo en los foros; indagar si el alcance y el sentido de este impacto difiere en función de la naturaleza de la información proporcionada -información de tipo estructural sobre el acceso, la participación y la conectividad en los foros o información sobre el contenido de las contribuciones de los participantes-; y verificar la utilidad de la analítica desarrollada para analizar los procesos de colaboración en línea.

La actividad de los participantes como fuente de información para promover la colaboración. Una analítica del aprendizaje basada en el modelo de Influencia Educativa Distribuida. César Coll, Anna Engel y Shamaly Niño.

Página 28 de 36. 
En lo que concierne al primer objetivo, los resultados muestran que el hecho de proporcionar información a los participantes sobre los componentes estructurales acceso, participación y conectividad- y sobre el contenido de sus contribuciones gestión de la participación, de la tarea y de los significados- tiene un impacto positivo inmediato y sostenido sobre el proceso colaborativo. En los cuatro grupos estudiados se constata en general un incremento en los valores de los indicadores grupales e individuales de acceso, participación y conectividad y en los indicadores grupales e individuales de gestión de la participación, de la tarea y de los significados. No se constata, sin embargo, una evolución clara en los valores de estos indicadores en los foros sucesivos pese a la presentación reiterada de información sobre la actividad desarrollada en el foro precedente a partir del F2.

En lo que concierne al segundo objetivo, los resultados obtenidos no apoyan la hipótesis de un impacto diferencial en función de la naturaleza de la información proporcionada a los grupos (G1: información estructural sobre acceso, participación y conectividad comunicada individualmente; G2: información sobre el contenido de las contribuciones comunicada individualmente; G3: ambos tipos de información comunicada individualmente; G4: información general sobre el cumplimiento de las directrices comunicada colectivamente). Como ya hemos mencionado, el hecho de proporcionar información sobre su actividad a los participantes al final del F1 provoca un aumento importante de los valores en los indicadores estructurales y de contenido en el F2 y siguientes en los cuatro grupos, sin que pueda apreciarse una diferencia nítida entre ellos en función de la naturaleza de la información proporcionada. El análisis comparativo detallado de los valores de los indicadores, en especial de los indicadores grupales e individuales relativos al contenido de las contribuciones, pone ciertamente de manifiesto la existencia de diferencias en los procesos colaborativos de los cuatro grupos, pero esas diferencias difícilmente pueden atribuirse a la naturaleza de la información proporcionada a los participantes de cada grupo. Recordemos brevemente las dos diferencias más importantes que hemos podido detectar mediante los análisis efectuados.

En primer lugar, si bien en los cuatro grupos se observa el mismo orden en los porcentajes de las contribuciones en las tres dimensiones, se constata también algunas diferencias importantes, especialmente en las dimensiones de participación y significados (ver Tabla 7). Así, el porcentaje de contribuciones en la dimensión de participación oscila entre el 2,57\% del G1 y el $12,11 \%$ del G3; y el porcentaje de contribuciones en la dimensión de significados entre el $70,29 \%$ del G1 y el $54,40 \%$ del G3. Estas diferencias, además, son consistentes, es decir, se mantienen a grandes trazos a través de los foros. En segundo lugar, si bien no hay diferencias entre los grupos en lo relativo a la presencia de las dos modalidades principales de ejercicio de la IE que hemos identificado (ver Tabla 9), sí que hemos observado diferencias en lo que concierne al impacto de las contribuciones de los participantes en las dimensiones

La actividad de los participantes como fuente de información para promover la colaboración. Una analítica del aprendizaje basada en el modelo de Influencia Educativa Distribuida. César Coll, Anna Engel y Shamaly Niño.

Página 29 de 36. 
de tarea y significados en la dinámica del proceso colaborativo en función de su peso relativo en las contribuciones del grupo en la dimensión correspondiente. Así, a grandes trazos, hemos visto que, mientras en G1 y G3 una mayoría de los sujetos realizan un número de contribuciones a las dimensiones de tarea, de significados o a ambas que se sitúa por encima de la mediana de las aportaciones de los miembros del grupo en la mitad o más de la mitad de los foros, en el G2 los sujetos que lo hacen son mayoritariamente los mismos en todos los foros y representan aproximadamente la mitad del grupo. A ello hay que añadir, además, la singularidad observada en los grupos G1 y G4, en los que encontramos participantes que suelen presentar un número de contribuciones que está por encima de la mediana de las contribuciones del grupo en una de las dos dimensiones, tarea o participación, pero no en la otra, lo que podría estar apuntando a la adopción por estos participantes de un rol focalizado en el ejercicio de la IE en la dimensión en cuestión.

Estas diferencias ponen de manifiesto la existencia de unas dinámicas de trabajo colaborativo en los grupos que van más allá de si los participantes ejercen o no la IE y si la ejercen o no en una u otra dimensión, y remiten más bien a cómo se distribuye el ejercicio de la IE que se lleva a cabo en el grupo. En algunos casos, por ejemplo, la distribución de la IE en el grupo puede adoptar la forma de una aportación equilibrada o relativamente equilibrada de la mayoría de los miembros del grupo al ejercicio de la IE en las tres dimensiones. En otros, en cambio, la distribución puede concretarse en que algunos miembros del grupo ejercen mayoritariamente la IE en unas dimensiones y otros en otras. De la misma manera que el modelo teórico de IE postula la existencia de modalidades distintas de ejercicio individual de la influencia educativa en función de la dimensión o dimensiones en que se ejerce de forma prioritaria, habría modalidades de distribución de la IE en los grupos en función de cuántos y cuáles de sus miembros contribuyen de forma relevante a ejercer la IE y en cuál o cuáles de las tres dimensiones se concentra este ejercicio. La hipótesis, entonces, es que para entender el impacto en el proceso de colaboración de la información proporcionada a los participantes sobre su actividad sería necesario tener en cuenta la dinámica grupal, y más concretamente la modalidad de distribución de la IE adoptada o en curso de adopción en el grupo, cuando se proporciona esta información a sus miembros. Aunque a nuestro entender algunos de los resultados expuestos apuntan en esta dirección, ni el diseño de la investigación realizada ni los datos obtenidos permiten un pronunciamiento claro al respecto. Para ello, será necesario llevar a cabo nuevas investigaciones en las que, además de la naturaleza de las informaciones proporcionadas a los participantes sobre su actividad, se preste especial atención a las modalidades de distribución del ejercicio de la IE.

Pero esto nos conduce directamente al tercer y último objetivo de nuestro trabajo relativo a la analítica empleada y a la adecuación y validez de los indicadores que propone para dar cuenta del ejercicio de la IE en los procesos de colaboración en línea. Los resultados obtenidos apoyan la afirmación de que la analítica utilizada ha

La actividad de los participantes como fuente de información para promover la colaboración. Una analítica del aprendizaje basada en el modelo de Influencia Educativa Distribuida. César Coll, Anna Engel y Shamaly Niño.

Página 30 de 36. 
permitido identificar, describir y analizar aspectos y componentes del desarrollo de los foros y de los procesos colaborativos generados en los grupos a los que difícilmente hubiéramos podido acceder sin su concurso. Junto a ello, sin embargo, ponen también de manifiesto algunas limitaciones y carencias de los indicadores utilizados y del propio modelo teórico de IED en los que se fundamentan. Permítasenos mencionar brevemente cuatro de ellas que nos parecen especialmente importantes por cuanto indican con claridad algunas vías de acción para seguir avanzando en el desarrollo y mejora del modelo.

En primer lugar, conviene precisar con mayor claridad de lo que hemos hecho hasta el momento (ver, por ejemplo, Coll, Bustos y Engel, 2011, 2015; Coll et al., 2013) la diferente naturaleza, alcance y valor de los indicadores estructurales y de contenido para dar cuenta de la IE ejercida por los participantes en un proceso colaborativo. Para decirlo en pocas palabras, los indicadores estructurales de acceso, participación y conectividad refieren a las condiciones para el ejercicio de la IE, mientras que los relativos al contenido de las contribuciones refieren al ejercicio mismo de la IE. Un participante con un índice individual de acceso elevado y un patrón individual de acceso continuo cumple dos condiciones favorables para el ejercicio potencial de la IE, pero que finalmente la ejerza o no, o que la ejerza de una u otra manera, no se puede saber hasta analizar el contenido de sus contribuciones, es decir, hasta que conozcamos sus índices individuales de gestión de participación, de tarea y de contenido.

En segundo lugar, y todavía en relación con los indicadores estructurales de acceso, participación y conectividad, en su versión actual el modelo de IED atribuye la misma importancia y valor a todos ellos como indicadores del cumplimiento o no cumplimiento de unas condiciones favorables para el ejercicio de la IE. Algunos resultados, sin embargo, sugieren que algunos indicadores tienen a este respecto mayor validez que otros. Así, por ejemplo, un índice individual de lectura elevado sitúa indudablemente al participante en una mejor situación para ejercer la IE que un índice individual de lectura bajo. En cambio, no es evidente que un índice individual bajo de reciprocidad sitúe necesariamente al participante en una posición de desventaja para ejercer la IE, ya que sus contribuciones pueden ser leídas por los otros participantes, y en consecuencia ejercer una IE, aunque no establezca relaciones recíprocas con ellos. Urge, en consecuencia, revisar la relevancia y validez de los indicadores estructurales propuestos por el modelo y otorgarles un peso diferencial en consecuencia como indicadores del cumplimiento de unas condiciones más o menos favorables para el ejercicio de la IE.

En tercer lugar, como ya hemos apuntado más arriba, es necesario elaborar y profundizar la idea ya apuntada de modalidades de distribución del ejercicio de la IE. Desde un punto de vista operacional, la IED ha sido concebida básicamente hasta el momento como el resultado de agregar las modalidades individuales de ejercicio de la

La actividad de los participantes como fuente de información para promover la colaboración. Una analítica del aprendizaje basada en el modelo de Influencia Educativa Distribuida. César Coll, Anna Engel y Shamaly Niño.

Página 31 de 36. 
IE de los miembros del grupo. Los análisis y resultados presentados indican, sin embargo, que las modalidades individuales de ejercicio de la IE no informan de forma fehaciente y fiable sobre el impacto de las contribuciones de los participantes en la dinámica de los procesos colaborativos ni sobre la distribución del ejercicio de la IE en el grupo. Urge, pues, desarrollar procedimientos e instrumentos que permitan identificar y describir las diferentes modalidades de distribución del ejercicio de la IE, y analizar el papel que juegan en la evolución y los resultados de los procesos de colaboración.

Y en cuarto y último lugar, los resultados presentados ponen de manifiesto que el ejercicio de la IE, y consecuentemente el uso y la utilidad de una analítica del aprendizaje inspirada en el modelo teórico de la IED, debe prestar una especial atención a las características y exigencias de la tarea y del contenido en torno al cual se articula el proceso colaborativo. El modelo de IED postula que, para que el proceso colaborativo fluya adecuadamente y su evolución y resultados sean satisfactorios, es necesario que se ejerza la IE en las tres dimensiones de gestión de la participación, de la tarea y de los significados. Ahora bien, como hemos visto, en los cuatro grupos estudiados la mayoría de las contribuciones de los participantes refieren a los significados sobre los contenidos de aprendizaje, un número importante pero sensiblemente menor de contribuciones refieren a la tarea, y un número realmente muy pequeño a la participación. Sería un error, sin embargo, valorar este reparto desigual de las contribuciones como una distribución inadecuada del ejercicio de la IE entre las tres dimensiones. La explicación hay que buscarla más bien en las características de la tarea y en las condiciones en que tiene lugar. Puesto que la tarea consiste en elaborar sendos glosarios de los conceptos más importantes de los núcleos temáticos, es lógico que la mayoría de las contribuciones tengan como foco los significados de los conceptos incluidos en los glosarios. Así mismo, puesto que las definiciones de los conceptos incluidos en los glosarios han de ser consensuadas por todos los miembros de cada grupo, es también lógico que un número significativo de contribuciones refiera al procedimiento a seguir o seguido para alcanzar dicho consenso. Y, por último, puesto que las directrices del profesor para la realización de los glosarios son muy estrictas en cuanto al número mínimo de contribuciones que deben realizar los miembros de los grupos, es igualmente lógico que las contribuciones relativas a la participación sean muy escasas.

Más allá del caso particular, estas consideraciones invitan a renunciar a la búsqueda de un modelo ideal de ejercicio de la IED para los procesos colaborativos, y por extensión de una analítica del aprendizaje inspirada en este modelo, con validez universal independientemente de las características concretas de estos procesos, sobre todo de las características de sus actores, de las tareas, contenidos y resultados en torno a los cuales se articula la colaboración y de las condiciones en las cuales se desarrollan. Una analítica del aprendizaje de este tenor tendría, a nuestro juicio, una utilidad muy limitada para apoyar y guiar los procesos interactivos entre estudiantes y

La actividad de los participantes como fuente de información para promover la colaboración. Una analítica del aprendizaje basada en el modelo de Influencia Educativa Distribuida. César Coll, Anna Engel y Shamaly Niño.

Página 32 de 36. 
enseñarles a participar, comunicarse, colaborar y aprender a través de la colaboración.

Presentación del artículo: 15 de Febrero de 2017

Fecha de aprobación: 4 de Marzo de 2017

Fecha de publicación: 31 de marzo de 2017

Coll, C.; Engel, A. y Niño, S. (2017). La actividad de los participantes como fuente de información para promover la colaboración. Una analítica del aprendizaje basada en el modelo de Influencia Educativa Distribuida. RED Revista de Educación a Distancia, 53. Consultado el (dd/mm/aaaa) en http://www.um.es/ead/red/53

\section{Referencias}

Banks, J., Au, K., Ball, A.F., Bell, P., Gordon, E., Gutierrez, K., .. Zhoum M.. (2007). Learning In and Out of School in Diverse Environments: Life-Long, Life-Wide, Life-Deep. The Learning in Informal and Formal Environments Center. University of Washington, Stanford University, and SRI International. Recuperado de http://life-slc.org/docs/Banks_etal-LIFE-Diversity-Report.pdf

Binkley, M., Erstad, O., Herman, J., Raizen, S., Ripley M., Miller-Ricci, M., \& Rumble, M. (2012). Defining Twenty First Century Skills. En P. Griffin, B. McGaw, \& E. Care (Eds.), Assessment and Teaching of 21st Century Skills (pp. 17-66). Dordrecht: Springer.

Borgatti, S.P., Everett, M.G., \& Freeman, L.C. (2002). Ucinet for Windows: Software for Social Network Analysis. Harvard, MA: Analytic Technologies.

Brown, M. (2012). Learning Analytics: Moving from concept to practice. Louisville, CO: EDUCAUSE Learning Initiative. Recuperado de http://net.educause.edu/ir/library/pdf/elib1203.pdf

Bustos, A. (2011). Presencia docente distribuida, influencia educativa y construcción del conocimiento en entornos de enseñanza y aprendizaje basados en la comunicación asíncrona escrita. Tesis doctoral. Universidad de Barcelona. Recuperado http://www.psyed.edu.es/prodGrintie/tesis/Tesis_A_Bustos_PresenciaDocenteD istribuida_V_web_2011.pdf

Bustos, A., Coll, C., y Engel, A. (2009). Presencia docente distribuida en redes asíncronas de aprendizaje. Definición teórica y perspectiva multimétodo para su estudio. En F. Díaz Barriga, G. Hernández, \& M. Rigo (Eds.), Aprender y enseñar con TIC en educación superior: contribuciones del socioconstructivismo. (pp. 97-128). México: Publicaciones Universidad Nacional Autónoma de México.

La actividad de los participantes como fuente de información para promover la colaboración. Una analítica del aprendizaje basada en el modelo de Influencia Educativa Distribuida. César Coll, Anna 
Coll, C. (2010). Enseñar y aprender, construir y compartir: procesos de aprendizaje y ayuda educativa. En C. Coll (Coord.). Desarrollo, aprendizaje y enseñanza en la Educación Secundaria (pp. 31-62). Barcelona: Graó

Coll, C., Bustos, A., y Engel, A. (2011). Perfiles de participación y presencia docente distribuida en redes asíncronas de aprendizaje: la articulación del análisis estructural y de contenido. Revista de Educación, 354, 657-688. Recuperado de http://www.mecd.gob.es/dctm/revista-de-educacion/articulosre354/re35426.pdf?documentld=0901e72b811e1d42

Coll, C., Bustos, A. y Engel, A. (2015). La información sobre el ejercicio de la influencia educativa como medio para favorecer la participación y el aprendizaje en un foro en línea. Infancia y Aprendizaje, 38, 368-401. http://dx.doi.org/10.1080/02103702.2015.1016745

Coll, C., Bustos, A., Engel, A., de Gispert, I., \& Rochera, M. J. (2013). Distributed Educational Influence and Computer-Supported Collaborative Learning. Digital Education Review, 24, 23-42. Recuperado de http://revistes.ub.edu/index.php/der/article/view/11274/pdf

Coll, C., Colomina, R., Onrubia, J., y Rochera, M. J. (1992). Actividad conjunta y habla: una aproximación a los mecanismos de influencia educativa. Infancia $y$ Aprendizaje, 59-60, 189-132. Recuperado de http://dialnet.unirioja.es/servlet/articulo?codigo $=48416$

Coll, C., \& Engel, A. (2014). Making meaning through joint activity in ComputerSupported Collaborative Learning (CSCL) settings: the interplay between content-related and activity-related talk. Anales de Psicología, 30(3), 818831. Recuperado de http://revistas.um.es/analesps/article/view/201181/164911

Coll, C., Engel, A., \& Bustos, A. (2009). Distributed Teaching Presence and Participants' Activity Profiles: a theoretical approach to the structural analysis of Asynchronous Learning Networks. European Journal of Education, 44(4), 521538.http://dx.doi.org/10.1111/j.1465-3435.2009.01406.x

Coll, C., Onrubia, J., y Mauri, T. (2008). Ayudar a aprender en contextos educativos: el ejercicio de la influencia educativa y el análisis de la enseñanza. Revista de Educación, 346, 33-70. Recuperado de http://www.revistaeducacion.mec.es/re346/re346_02.pdf

Colomina, R., Onrubia, J., y Rochera, M. J. (2001). Interactividad, mecanismos de influencia educativa y construcción del conocimiento en el aula. En C. Coll, J. Palacios y A. Marchesi (Comps.). Desarrollo Psicológico y educación. Vol. II. Psicología de la Educación (pp. 415-435). Madrid: Alianza.

Dawson, S., Gašević, D., Siemens, G., \& Joksimovic, S. (2014). Current State and Future Trends: A Citation Network Analysis of the Learning Analytics Field. En Proceedings of the Fourth International Conference on Learning Analytics And

La actividad de los participantes como fuente de información para promover la colaboración. Una analítica del aprendizaje basada en el modelo de Influencia Educativa Distribuida. César Coll, Anna Engel y Shamaly Niño.

Página 34 de 36. 
Knowledge (pp. 231-240). New York, NY, USA: ACM. http://dx.doi.org/10.1145/2567574.2567585

Dawson, S., \& Siemens, G. (2014). Analytics to Literacies: The development of a learning analytics framework for multiliteracies assessment. The International Review of Research in Open and Distance Learning, 15(4), 284-305. Recuperado de http://www.irrodl.org/index.php/irrodl/article/view/1878/3006

Engel, A., Coll, C., \& Bustos, A. (2013). Distributed Teaching Presence and communicative patterns in asynchronous learning: Name versus reply networks. Computers \& Education, 60, 184-196. http://dx.doi.org/10.1016/j.compedu.2012.06.011

Ferguson, R. (2012). Learning analytics: drivers, developments and challenges. International Journal of Technology Enhanced Learning, 4(5/6), 304-317. http://dx.doi.org/10.1504/IJTEL.2012.051816

Fidalgo-Blanco, Á., Sein-Echaluce, M.L., García-Peñalvo, F.J., \& Conde, M. Á. (2015) Using Learning Analytics to improve teamwork assessment. Computers in Human Behavior.47, 149-156. http://dx.doi.org/10.1016/j.chb.2014.11.050

Haya, P. A., Daems, O., Malzahn, N., Castellanos, J., \& Hoppe, H. U. (2015). Analysing content and patterns of interaction for improving the learning design of networked learning environments. British Journal of Educational Technology, 46(2), 300-316. http://dx.doi.org/10.1111/bjet.12264

Jenkins, H., Clinton, K., Purushotma, R., Robinson, A. J., \& Weigel, M. (2006). Confronting the challenges of participatory culture: Media education for the 21st century. Chicago, IL: MacArthur Foundation.

Mazza, R., \& Dimitrova, V. (2007). CourseVis: A graphical student monitoring tool for supporting instructors in Web-based distance courses. International Journal of Human-Computer Studies, 65(2), 125-139. http://dx.doi.org/10.1016/j.ijhcs.2006.08.008

Mercer, N. (1995) The Guided Construction of Knowledge: talk amongst teachers and learners. Clevedon: Multilingual Matters.

Rodríguez-Triana, M. J., Martínez-Monés, A., Asensio-Pérez, J. I., \& Dimitriadis, Y. (2015). Scripting and monitoring meet each other: Aligning learning analytics and learning design to support teachers in orchestrating CSCL situations. British Journal of Educational Technology, 46(2), 330-343. http://dx.doi.org/10.1111/bjet.12198

Romero, C., Ventura, S., \& García, E. (2007). Data mining in course management systems: Moodle case study and tutorial. Computers \& Education, 51(1), 368384. http://dx.doi.org/10.1016/j.compedu.2007.05.016

Roschelle, J. (2013). Special issue on CSCL: discussion. Educational Psychologist,

La actividad de los participantes como fuente de información para promover la colaboración. Una analítica del aprendizaje basada en el modelo de Influencia Educativa Distribuida. César Coll, Anna Engel y Shamaly Niño.

Página 35 de 36. 
48(1), 67-70. http://dx.doi.org/10.1080/00461520.2012.749445

Siemens, G. \& Long, P. (2011). Penetrating the Fog: Analytics in learning and Education. EDUCAUSE Review 46(5), 30-40. Recuperado de http://www.educause.edu/ero/article/penetrating-fog-analytics-learning-andeducation

Stahl, G., Koschmann, T., \& Suthers, D. (2006). Computer-supported collaborative learning: An historical perspective. In R. K. Sawyer (Ed.), Cambridge handbook of the learning sciences (pp. 409-426). Cambridge, UK: Cambridge University Press. Retrieved from: http://GerryStahl.net/cscl/CSCL_English.pdf

Suthers, D. D., \& Verbert, K. (2013). Learning analytics as a "middle space." En Proceedings of the Third International Conference on Learning Analytics and Knowledge (pp. 1-4). New York, NY, USA: ACM. http://dx.doi.org/10.1145/2460296.2460298

Valentín, L. de la F., \& Burgos, D. (2015). A4Learning: An Iterative Methodological Approach to Support Better Learning and Teaching. IEEE LATIN AMERICA TRANSACTIONS, 13(2), 483-489. http://dx.doi.org/10.1109/TLA.2015.7055568

Wilson, M., \& Scalise, K. (2015). Assessment of Learning in Digital Networks. En P. Griffin \& E. Care (Eds.). Assessment and Teaching of 21st Century Skills: Methods and Approaches (pp. 57-81). Dordrecht: Springer.

Xing, W., Guo, R., Petakovic, E., \& Goggins, S. (2015). Participation-based student final performance prediction model through interpretable Genetic Programming: Integrating learning analytics, educational data mining and theory. Computers in Human Behavior, 47, 168-181. http://dx.doi.org/10.1016/j.chb.2014.09.034

Yin, R. K. (2006). Case Study methods. En J. L. Green, G. Camilli \& P. B. Elmore (Eds.), Handbook of Complementary Methods in Education Research (pp. 111 -122). Mahwah, NJ: L. Erlbaum.

Zapata-Ros, M. (2013). Analítica del aprendizaje y personalización. Campus virtuales, 2(2), 88-119. Recuperado de http://www.uajournals.com/campusvirtuales/journal/3/7.pdf

La actividad de los participantes como fuente de información para promover la colaboración. Una analítica del aprendizaje basada en el modelo de Influencia Educativa Distribuida. César Coll, Anna Engel y Shamaly Niño. 